\title{
Conformation Manipulation and Motion of a Double Paddle Molecule on an Au(111) Surface
}

\author{
We-Hyo Soe, ${ }^{1, *}$ Yasuhiro Shirai, ${ }^{2}$ Corentin Durand, ${ }^{1}$ Yusuke Yonamine, ${ }^{3, \dagger}$ Kosuke Minami, ${ }^{3}$ \\ Xavier Bouju, ${ }^{1}$ Marek Kolmer, ${ }^{4}$ Katsuhiko Ariga, ${ }^{3,5}$ Christian Joachim,,${ }^{1,3}$ and Waka Nakanishi ${ }^{3, \ddagger}$ \\ ${ }^{1}$ GNS and MANA Satellite, CEMES-CNRS, \\ 29 Rue J. Marvig, BP 4347, 31055 Cedex Toulouse, France. \\ ${ }^{2}$ GREEN, National Institute for Materials Science, \\ 1-1 Namiki, Tsukuba, Ibaraki 305-0044, Japan. \\ ${ }^{3}$ WPI-MANA, National Institute for Materials Science, \\ 1-1 Namiki, Tsukuba, Ibaraki 305-0044, Japan. \\ ${ }^{4}$ Centre for Nanometer-Scale Science and Advanced Materials, \\ NANOSAM, Faculty of Physics, Astronomy, \\ and Applied Computer Science, Jagiellonian University, \\ Łojasiewicza 11, PL 30-348 Krakow, Poland. \\ ${ }^{5}$ Graduate School of Frontier Sciences, \\ The University of Tokyo, Kashiwa 277-0827, Japan.
}




\begin{abstract}
The molecular conformation of a bisbinaphthyldurene (BBD) molecule is manipulated using a lowtemperature ultrahigh-vacuum scanning tunneling microscope (LT-UHV STM) on an Au(111) surface. BBD has two binaphthyl groups at both ends connected to a central durene leading to anti/syn/flat conformers. In solution, dynamic nuclear magnetic resonance indicated the fast interexchange between the anti and syn conformers as confirmed by density functional theory calculations. After deposition in a submonolayer on an $\mathrm{Au}(111)$ surface, only the syn conformers were observed forming small islands of self-assembled syn dimers. The syn dimers can be separated into syn monomers by STM molecular manipulations. A flat conformer can also be prepared by using a peculiar mechanical unfolding of a syn monomer by STM manipulations. The experimental STM $d I / d V$ and theoretical elastic scattering quantum chemistry maps of the low-lying tunneling resonances confirmed the flat conformer BBD molecule STM production. The key BBD electronic states for a step-by-step STM inelastic excitation lateral motion on the Au(111) are presented requiring no mechanical interactions between the STM tip apex and the BBD. On the BBD molecular board, selected STM tip apex positions for this inelastic tunneling excitation enable the flat BBD to move controllably on $\mathrm{Au}(111)$ by a step of $0.29 \mathrm{~nm}$ per bias voltage ramp.
\end{abstract}


With the tip of a scanning tunneling microscope (STM), atomic-scale manipulation protocols are well-known since the pioneering work of D. Eigler, ${ }^{1}$ and precise studies have described the various mechanisms of single atom (a small molecule) mechanical manipulations. ${ }^{2,3}$ Pushing a single large molecule on a surface with the tip of the $\mathrm{STM}^{4,5}$ is now a standard procedure to position precisely functioning molecules on a surface for single molecule mechanics experiments ${ }^{6}$ and also for single molecule electronic measurements. ${ }^{7}$

To perform an atomically precise lateral manipulation of a molecule on a metallic surface with no mechanical interactions between the STM tip apex and the molecule, the bias tip must be able to feed up energy to the molecule with a few picometer lateral precision. ${ }^{8}$ This excitation can be either inelastic from the tunneling current itself or originate from the enhanced electric field located in the biased tip/surface junction when the molecule carries a local dipolar moment. ${ }^{9}$ For inelastic tunneling excitations, the energy entry port is generally the low-lying reduced electronic states of the molecule. ${ }^{7,10}$ Here, a precise design of the molecule is required to avoid the energy provided by the tunneling current passing through the molecule from being equally distributed among the many mechanical degrees of freedom of this molecule. If not, a conformation change of the molecule may happen but with no lateral displacements. The molecule can also be broken in small chemical groups by the applied bias voltage pulse ${ }^{11}$ instead of moving step-by-step on the supporting surface by steps, generally the commensurable surface atomic lattice constant. To also avoid energy redistribution toward the supporting surface, different leg and wheel molecular groups have been early identified. They can efficiently maintain a space (van der Waals distances) between the planar molecular chassis and the supporting surface. ${ }^{12-14}$ Due, for example, to steric crowding, lateral chemical groups not having the shape of a leg or a wheel, mounted on the chassis in a symmetric way and holding it at van der Waals distances from the surface, are also interesting for molecular design as presented in this paper. ${ }^{15}$ The light-driven molecular motor of the Feringa group the first switchable chemical group to be mounted by the Tour group on a chassis equipped with four wheels ${ }^{16}$ in an attempt to leave space for this molecular group to change its conformation/configuration using an optical excitation. ${ }^{17} \mathrm{~A}$ similar molecular switch was used by the Feringa group to obtain a molecule with four of those, used as switchable legs under a tunneling inelastic excitation. ${ }^{18}$ Other switchable chemical groups are also available for equipping a molecular chassis. For example, molecules carrying a photoisomerizable double bond, such as stilbene, azobenzene, or diarylethene, have been used as molecular switches. ${ }^{19}$ Their photoisomerization is usually studied in the gas phase or in solution. Conformation/configuration change 
triggered by tunneling electrons has also been observed in STM single molecule experiments, like with azobenzene. ${ }^{20-22}$ Other molecules are also available which can twist around a single bond by photoirradiation. Twisted intramolecular charge transfer (TICT) molecules provide a nice example of such a light-activated conformation change. ${ }^{23}$

Binaphthyl molecules or their derivatives (Scheme ) belong to another group of photosensitive compounds which are also known to change conformation under UV irradiation. ${ }^{24,25}$ In this paper, we present the design and synthesis of a bisbinaphthyldurene (BBD) molecule (Scheme) for STM imaging, single molecule manipulation, and step-by-step lateral motions. This molecule is equipped with two binaphthyl paddles mounted laterally on a very simple central phenyl chassis. On a planar BBD, we demonstrate here how to use the low amplitude vibration modes of its 1,1'-binaphthyl lateral paddles ${ }^{26,27}$ for manipulating the BBD along a $\mathrm{Au}(111)$ surface using STM inelastic tunneling effects. Not existing in solution, this planar conformation is stabilized by the $\mathrm{Au}(111)$ surface. On $\mathrm{Au}(111)$, it enters in competition with its native in solution nonplanar conformation which can also be reached by the same excitation on a metallic surface as presented below.

In the initial subsections of the Results and Discussion, the design, the synthesis, and the structural analysis of the BBD molecules in solution are provided together with a detailed DFT theoretical study of the different possible conformations of a BBD molecule. In the following subsections, STM images of the BBD molecules on the $\mathrm{Au}(111)$ surface acquired at low-temperature (LT) and in ultrahigh-vacuum environment (UHV) are provided. We demonstrate how to prepare the BBD molecule in a planar conformation on the $\mathrm{Au}(111)$ surface using a very specific STM tip lateral molecular manipulation protocol. In this planar conformation, the BBD electronic probability density map of its electronic states around the $\mathrm{Au}(111)$ surface Fermi level can be recorded to prepare the BBD inelastic manipulation. In the final subsection, the entry ports for tunneling electron energy transfer to the BBD molecule are identified. It is shown how to STM manipulate step-by-step the BBD molecule by step of $0.29 \mathrm{~nm}$ on the $\mathrm{Au}(111)$ surface. 


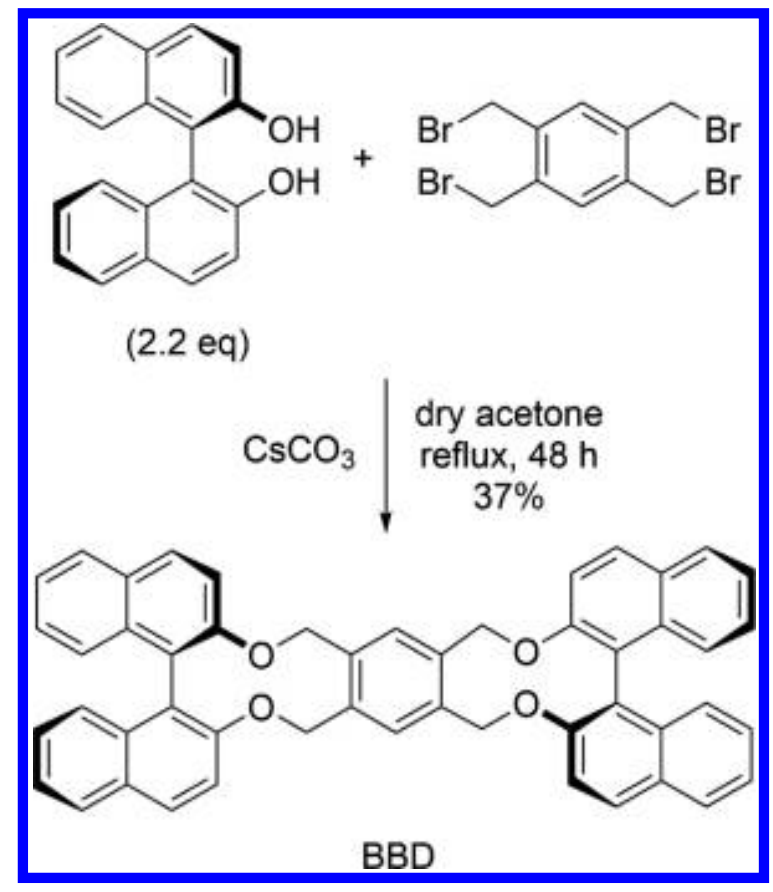

Scheme 1. Synthetic Route of BBD.

\section{RESULTS AND DISCUSSIONS}

\section{A. Design and Chemical Synthesis}

A 1,1'-binaphthyl molecule consists of two naphthalene moieties with one single phenyl per moiety connected via a $\mathrm{C}-\mathrm{C}$ single bond. The distinct characteristics of a 1,1'-binaphthyl are (1) its flexibility around this $\mathrm{C}-\mathrm{C}$ bond ${ }^{24,25,28-30}$ and (2) the axial chirality originating from the inhibition by steric crowding of a complete $360^{\circ}$ naphthyl-naphthyl rotation around its joint $\mathrm{C}-\mathrm{C}$ bond. ${ }^{24,25,28-30}$ The enantiomers of axially chiral compounds are classified using the stereochemical labels $R$ and $S$ based on their absolute configuration around a stereocenter. Chiral 1,1'- binaphthyls and derivatives having a naphthyl torsion angle between $-180^{\circ}<\theta<0^{\circ}$ correspond to the $R$ configurations and those between $0^{\circ}<\theta<180^{\circ}$ correspond to the $S$ configurations, respectively. In its $S_{0}$ electronic ground state and as a function of the torsion angle $|\theta|$, the 1,1'-binaphthyl conformation angle can vary from $60^{\circ}$ to $120^{\circ}$ within $<1 \mathrm{kcal} / \mathrm{mol}$ of energy. The potential energy 
curve along this conformation change is a flat-bottomed well where the $|\theta| \sim 90^{\circ}$ saddle point separates two shallow wells whose minima are at $|\theta| \sim 70^{\circ}$ and $|\theta| \sim 110^{\circ} .^{24,25,28-30}$ Since the conformation of chiral binaphthyls can be monitored by circular dichroism (CD) spectra, conformation controllability in this ground state was demonstrated at the air-water interface by applying a small mechanical force. ${ }^{31,32}$ The difference between the $S_{0} 1,1$ '- binaphthyl relaxed conformations and the $\mathrm{S}_{1}$ lowest excited singlet state conformations ${ }^{26,27}$ is at the origin of our BBD internal mechanical vibrations because of the reversal in $\theta$ of the $S_{1}(\theta)$ relative to the $S_{0}(\theta)$ double well potential energy curve minima. ${ }^{26,27}$ Although for cisoid $\left(|\theta|<90^{\circ}\right)$ and transoid $\left(|\theta|>90^{\circ}\right)$, the $S_{1}$ and $S_{0}$ relaxed conformations are still under discussion, and this difference was important to preserve in the BBD design. For example, time-dependent density functional theory (TD-DFT) calculations show that the $(R)-1,1$ '-bi(2-naphthol) molecule (the starting compound for the BBD synthesis; Scheme ) still preserves a different relaxed conformation between $\mathrm{S}_{0}\left(\theta=-91^{\circ}\right)$ and $\mathrm{S}_{1}$ $\left(\theta=-119^{\circ}\right)$ that triggers an almost $30^{\circ}$ paddle effect going back and forth optically from $\mathrm{S}_{0}$ to $\mathrm{S}_{1}$ (Figure S16 in the Supporting Information).

Entering now in the design of our molecule, two binaphthyl molecules are used in the BBD as lateral paddles because of this reversal of the relative minimum energy $|\theta|$ value between $S_{1}$ and $\mathrm{S}_{0}$ and because of the low $1 \mathrm{kcal} / \mathrm{mol}$ energy barrier between the two minima in the $\mathrm{S}_{0}$ ground state. The two binaphthyls are connected laterally to a very small chassis made simply of a central phenyl (BBD in Scheme 1). This covalent binding of each binaphthyl via the methylene oxy bridges modifies the paddle switch ability with, for example, the suppression of the $S_{1}\left(\theta=-119^{\circ}\right.$ ) torsion angle energy minimum. What is important here is that $S_{0}$ keeps its awaited mechanical characteristics, that is, the possibility of its vibrational oscillations around its new $\left(\theta=-61^{\circ}\right)$ ground-state minimum (TD-DFT calculated) reachable, for example, by optical excitation and relaxation via its new $\mathrm{S}_{1}\left(\theta=-58^{\circ}\right)$ relaxed conformation for the $(R, R)$ isomer (Figure $\mathrm{S} 17$ in the Supporting Information).

On a metallic surface and in a planar conformation, the two BBD binaphthyl groups permit to space the BBD chassis away from this surface at a distance compatible with a physisorption state. To drive the BBD molecule step-by-step along an fcc track of the Au(111) surface using the inelastic effect of the STM tunneling current, one has to first virtually prepare this molecule in its instantaneous virtual reduced electronic state well described for its mechanics by considering in first approximation the BBD $\mathrm{S}_{1}$ excited state. Afterward, relaxation to the $\mathrm{S}_{0}$ ground state will result in a small amplitude and noncoherent binaphthyl oscillations. As a function on the tip apex 
location on $\mathrm{BBD}$, this will generate a surface lateral motion over the lateral diffusion barrier of the $\mathrm{Au}(111)$ fcc portion of the herringbone surface reconstruction.

The BBD molecule was synthesized by a one-pot reaction from commercially available $(R)$ 1,1'-bi(2-naphthol) and $\alpha, \alpha^{\prime}, \alpha^{\prime \prime}, \alpha^{\prime \prime \prime}$-tetrabromodurene (Scheme 1). Before its evaporation in the STM preparation chamber, it was further ultrapurified by sublimation to produce a colorless powder with no crystallinity. BBD UV-vis absorption spectrum is similar to the one of binaphthyl molecules $^{33}$ and shows an absorption peak maximum at $334 \mathrm{~nm}$ (Figure S1 in the Supporting Information), demonstrating a good electronic separation between the two BBD lateral paddles. Recorded conventional CD spectra of chiral binaphthyls (Figure S2 in the Supporting Information) confirmed that the chirality of the binaphthyl groups remained after purification.

\section{B. The Native BBD Molecule Conformation in Solution.}

Variable-temperature (VT) analysis with NMR spectroscopy (Figure S13 in the Supporting Information) revealed the dynamic fluctuations between two BBD conformers with the same equilibrium population in solution. The two sets of ${ }^{1} \mathrm{H}$ NMR peaks that originate from those two conformers were also observed at low temperature from 218 to $223 \mathrm{~K}$ showing no sign of a favored conformer (Figure S13 in the Supporting Information). The analysis of those VT NMR spectra by a line- shape-fitting provides the experimental energetics for the interexchange processes between the two BBD conformers. Using an Eyring plot, the parameters of this interexchange were estimated to be $\Delta H=9.8 \mathrm{kcal} / \mathrm{mol}$ and $\Delta S=-17 \mathrm{cal} /(\mathrm{mol} \mathrm{K}$ ) (Figures $\mathrm{S} 14$ and $\mathrm{S} 15$ in the Supporting Information), supporting the possibility of a fast interconversion of the two conformers at ambient temperature and in solution.

As presented in Figure 1, three BBD conformers were identified using DFT calculations (B3LYP/6-31G(d,p)) depending on the location of the two binaphthyl paddles relative to the central phenyl. They correspond to the flat, syn, and anti conformations of a BBD molecule. The syn and anti conformers are expected to be the principal BBD isomers in solution since after molecular structure optimization, the flat, syn, and anti relative conformation energies are 20, 0.4, and $0.0 \mathrm{kcal} / \mathrm{mol}$, respectively. Experimental ROESY peaks in NMR spectroscopy are also consistent with the existence of the anti-syn conformer in solution since a weak correlation was observed between the central aromatic $\mathrm{CH}$ and the side binaphthyl aromatic $\mathrm{CH}$ proton (Figures S10-S12 in the Supporting Information). 


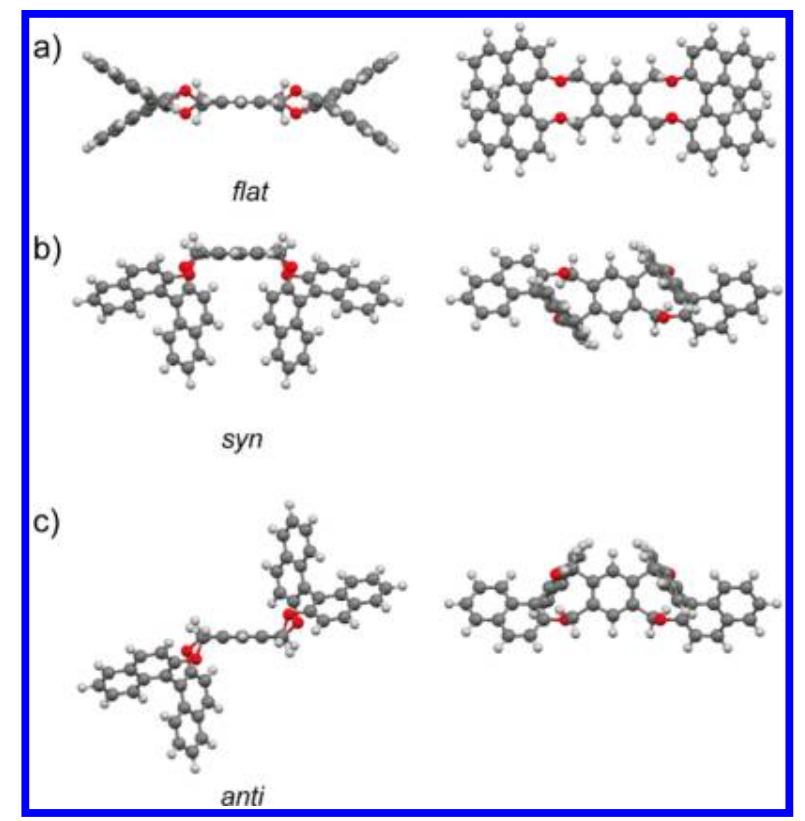

Figure 1. Various possible conformers of BBD molecule. (a) Flat, (b) syn, and (c) anti conformers obtained from DFT calculations $(B 3 L Y P / 6-31 G(d, p))$ with relative energies of +20 ,

+0.4 , and $0.0 \mathrm{kcal} / \mathrm{mol}$, respectively. Each structure was optimized with its $D_{2}, C_{2}$, and $C_{2}$ symmetry, respectively. The calculated torsion angles of the binaphthyl are $\theta=-61^{\circ},-64^{\circ}$ and $-64^{\circ}$, respectively. In solution, only the anti and syn conformers have been identified. Physisorbed on an Au(111) surface, STM molecular manipulations of BBD lead to the production of the flat conformer.

A flat BBD conformer is supposed by design to render accessible the different entry ports on its board for local STM excitations. In the following section, we will demonstrate how this flat conformer can be produced molecule per molecule by STM single molecule mechanical manipulations. When obtained, this flat conformer turns out to be quite stable on the Au(111) surface. 


\section{Native BBD Conformation and 2D Organization on the Au(111) Surface.}

Two typical constant current STM images obtained after BBD molecules deposition on the $\mathrm{Au}(111)$ reconstructed surface are presented in Figure 2. They were mainly found self-assembled in small 2D islands (Figure 2a). In some place, single BBD molecule lines can also be observed. This pseudo-1D growth along the Au(111) herringbone track is usually stopped at both ends of the line by a different surface BBD molecular ordering (Figure 2b). In all those observed pseudo-1D and 2D surface molecular orderings, the BBD molecules appear having the shape of a curve letter " $f$ ". Those " $f$ " BBD molecules have three possible adsorption directions on the Au(111) surface (see the Figure 2a insert). As presented in Figure 2b, the observed single " $f$ " BBD lines confirm that the BBD molecules are sensitive to the lateral ridges of the herringbone reconstruction (in average $0.03 \mathrm{~nm}$ in height). ${ }^{6}$

As certified experimentally by STM single molecule manipulations in the next subsection, each "f" STM molecular feature is a BBD dimer consisting of two syn conformers oriented perpendicular to the surface plane. They are coupled by a pair along one of the three [211] crystallographic orientations of the $\mathrm{Au}(111)$ fcc portion of this surface (see the Figure 2a insert). A first experimental indication of this pairing is evidenced in Figure 2 a by analyzing the only molecular alignment defect at the top of the last left BBDs molecular row.

As discussed in the previous subsection, the BBD molecules are found equally in the syn and anti conformations in solution. Molecular dynamics (MD) simulations were performed to simulate a hot adsorption process of the BBD molecules on a $\mathrm{Au}(111)$ surface (see Supporting Information). When the BBD molecules are annealed on the surface up to $500 \mathrm{~K}$, the syn and anti conformers are deformed but remain on the surface with no transformation in the flat conformer. At this temperature and during their 2D diffusion around the $\mathrm{Au}(111)$ surface, the BBD molecules have enough kinetic energy to mutually transform between the syn and anti as they certainly performed in solution and at room temperature. Upon cooling down the surface to room temperature, the BBD molecules thermalize toward the syn conformers since syn is $9.0 \mathrm{kcal} / \mathrm{mol}$ lower in energy as compared to anti on the $\mathrm{Au}(111)$ surface. Furthermore, the syn thermalize with still their central phenyl perpendicular to the $\mathrm{Au}(111)$ surface because laterally stabilized by their two paddles. During a slow thermalization process, the syn will also continue to diffuse on the surface. While in this perpendicular adsorption conformation, they can pair via a central phenyl $\pi$-stacking inter-

actions as also confirmed by MD calculation (see Supporting Information). It results in the " $f$ " 


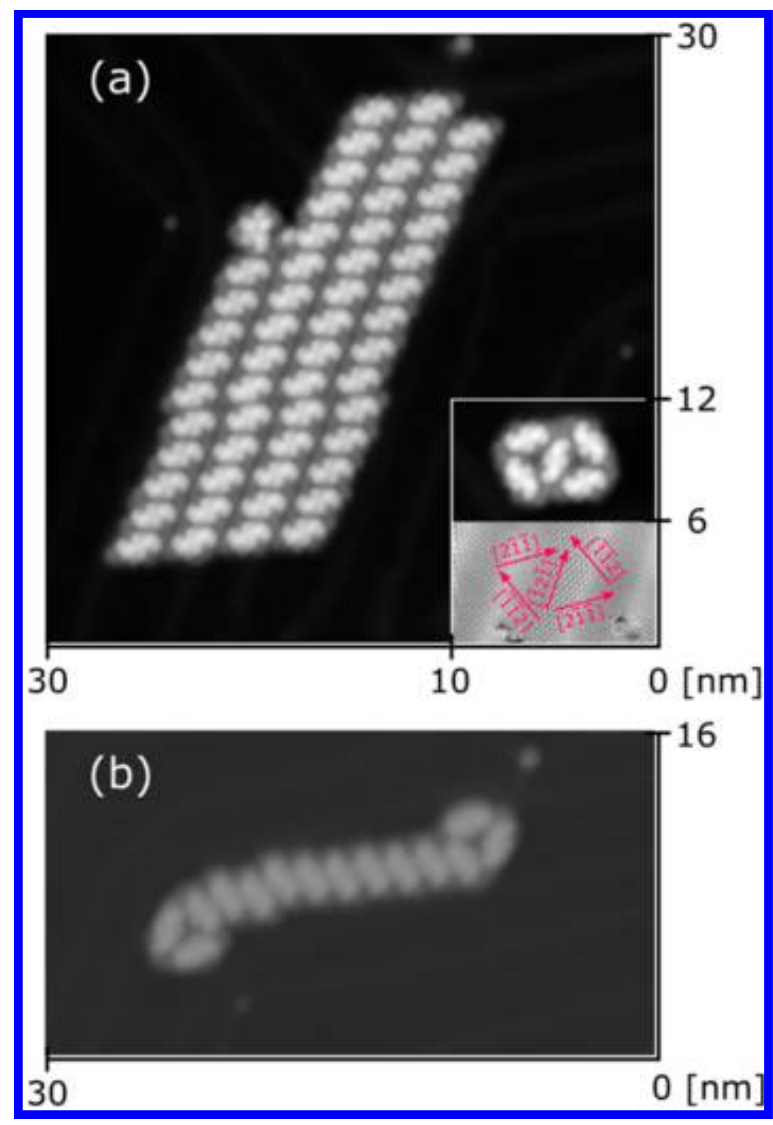

Figure 2. (a) A typical STM topographic image of BBD molecules forming a small 2D island on the $A u(111)$ reconstructed surface. Each building block of the imaged island is a dimer of syn-syn conformers having the shape of a curved " $f$ " letter. Insert a: the three possible adsorption orientations of the syn dimers. The atomic resolved image was also recorded using a molecule terminated STM tip to confirm the molecular orientation. (b) A good example of supramolecular assembly with a line of $10 \mathrm{BBD}$ molecular dimers formed along an Au(111) herringbone whose growth was stopped at both ends of the BBD line by 2 " $f$ " dimers of a different surface orientation. (All LT-UHV STM constant current image images were generally recorded at

$$
I=20 p A, V=0.5 \text { V.) }
$$


STM image like shape dimers observed in Figure 2 assembled in the various pseudo-1D line and $2 \mathrm{D}$ islands.

According to MD calculations and if accessible, the flat BBD conformers would be more stable than the orthogonal to the surface syn and anti conformers. However, getting directly a flat conformer from the native syn and anti conformers on the $\mathrm{Au}(111)$ surface would require an annealing temperature of about $1500 \mathrm{~K}$. Therefore, during deposition or by heating up the surface directly afterward, it will be difficult to produce flat conformers. At such a high temperature, most of the BBD molecules will break and/or desorb from the surface. Anyhow, the gold melting point is lower than $1500 \mathrm{~K}$. As demonstrated below, flat conformers can be produced molecule per molecule on the $\mathrm{Au}(111)$ surface starting from the orthogonal to the surface syn conformers using a very specific STM single molecule mechanical manipulation protocol.

\section{STM Single Molecule Mechanical Manipulation for Preparing flat BBD Conformers.}

To produce a flat BBD conformer, a selected syn conformer dimer adsorbed perpendicular to the $\mathrm{Au}(111)$ surface (one of the " $f$ " molecular units imaged in Figure 2) must be first separated into independent syn monomers. For this purpose and starting from a 2D island of the sort imaged in Figure 2a, STM lateral BBD molecule mechanical manipulation has been first performed as presented in Figure 3. Here, the threshold STM tunneling resistance for molecule manipulation is around $R_{T}=270 \mathrm{M} \Omega$. In most cases, a " $f$ " dimer can readily be separated out of the 2D island but only as a single " f" dimer entity with no monomer separation as presented in the Figure 3a,b. Then, a " $f$ " dimer can be step-by-step displaced over quite long distances over the surface in such STM manipulation conditions. When they are sometimes disassembled into two syn monomers

during this process, one syn of the " $f$ " pair is generally transferred to the STM tip, and the other one remains in the island as shown in the sequence Figure 3b,c.

Notice also that after the breaking of a " $f$ " pair at the 2D island border and in its orthogonal to the surface adsorption configuration, the syn monomer left in the island (as obtained in Figure 3c) can be further extracted from this island byr a further lateral STM manipulation. In this case, it has also a high probability to be captured by the tip apex, confirming how this syn monomer orthogonal configuration is not very stable on an $\mathrm{Au}(111)$ surface. We have succeeded to manipulate a few of those syn monomers toward specific $\mathrm{Au}(111)$ surface areas like the herringbone kinks where generally the surface atomic order is not regular and can stabilize them. They can also be dragged 


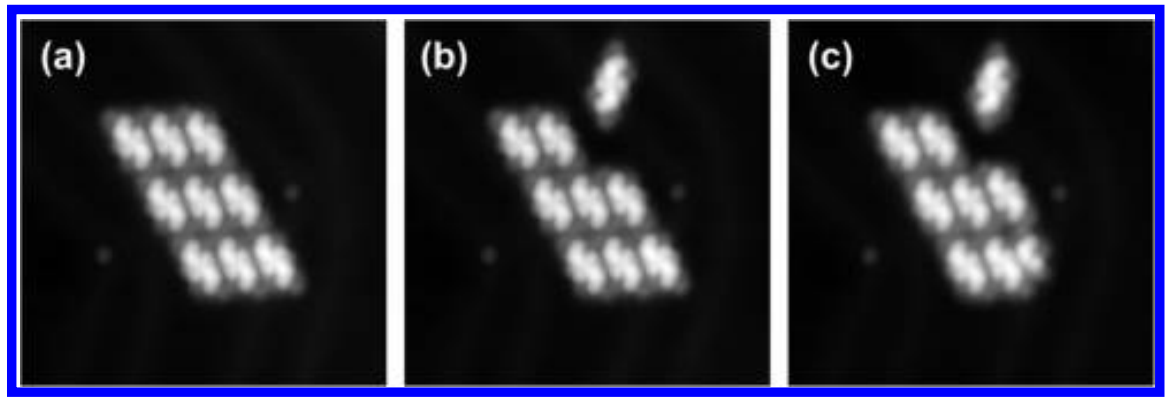

Figure 3. An example of the molecule manipulation experiments using the tunneling condition near the threshold $R_{T}=270 \mathrm{M \Omega}$. (a) $2 \mathrm{D}$ island of self-assembled syn-syn dimers on the Au(111) surface observed just after Au(111) sample preparation. (b) A " $f$ ” BBD molecule was step-by-step manipulated and extracted from its island while maintaining its dimer structure. (c) A monomer was detached from a down right corner " $f$ " of the island and adsorbed to the tip apex and remains of the second syn of this dimer initially perpendicular to the surface conformation (same STM image conditions as in Figure 2. Image size: $15 \mathrm{~nm} \times 15 \mathrm{~nm}$ ). 
along the surface during standard imaging conditions, that is, for STM $R_{T}$ around $10 \mathrm{G} \Omega$ and a tunneling current below $10 \mathrm{pA}$.

At the border of a 2D-island and using $R_{T} \sim 120 \mathrm{M} \Omega$, a new specific BBD dimer molecule

manipulation protocol can bring a different manipulation outcome. When in a " $f$ " pair located at the border of a 2D island, a syn BBD molecule is manipulated without trying to extract it directly from this island border; however, following the appropriate manipulation tip trajectory presented in Figure 4, a flat monomer can be produced with its two-fold paddles now fully open. Figure 4 presents an example of such an outcome with a reasonable $10 \%$ probability of success.

There are two essential conditions for this specific protocol to produce successfully a flat conformer and to open the BBD two paddles. First, the syn targeted BBD molecule must be paired with a syn $\mathrm{BBD}$ anchored at the edge of a $2 \mathrm{D}$ island but not at a corner. This anchoring will serve as a pivot for the opening following a classical molecular mechanical motion of the molecule, as if it was a solid and rigid body pivoting around a fix point. Second, the targeted syn BBD must be "rubbed" laterally on another " $f$ " dimer of the island during the manipulation, that is, the manipulation trajectory must maintain a lateral interaction with the other " $f$ " dimer for the flat flipping of the manipulated BBD molecule to be complete. As a consequence, the manipulated BBD molecule performs a $90^{\circ}$ flip down to the surface to reach a planar central phenyl configuration with the two paddles opened flat on the $\mathrm{Au}(111)$ surfacem as illustrated in Figure $4 a, b$ (more examples in Supporting Information section 9). In this case, the lateral required interactions between the manipulated BBD and the border 2D island BBDs seem to be attractive according to the recorded manipulation signal, but this requires a more detailed interpretation in the future.

When produced, a flat conformer is very stable on the $\mathrm{Au}(111)$ surface as theoretically predicted by MD calculations. After its production, this is confirmed by the experimental RT threshold value for a flat conformer STM molecular manipulation in a pushing mode along the $\mathrm{Au}(111)$ surface, the lowest ( $\sim 66 \mathrm{M} \Omega$ ) of all the $R_{T}$ values used for the different BBD molecule configurations met on this surface. A flat BBD monomer can be truly and reproducibly STM manipulated mechanically over long distances as presented in Figure 4b,c.

\section{E. Tunneling Spectroscopy and States Mapping of the Flat BBD Conformer.}

A flat BBD conformer on the $\mathrm{Au}(111)$ surface opens access to the detail STM $\mathrm{d} I / \mathrm{d} V$ mapping of its low-lying molecular electronic states around the Au(111) surface Fermi energy. Such a 


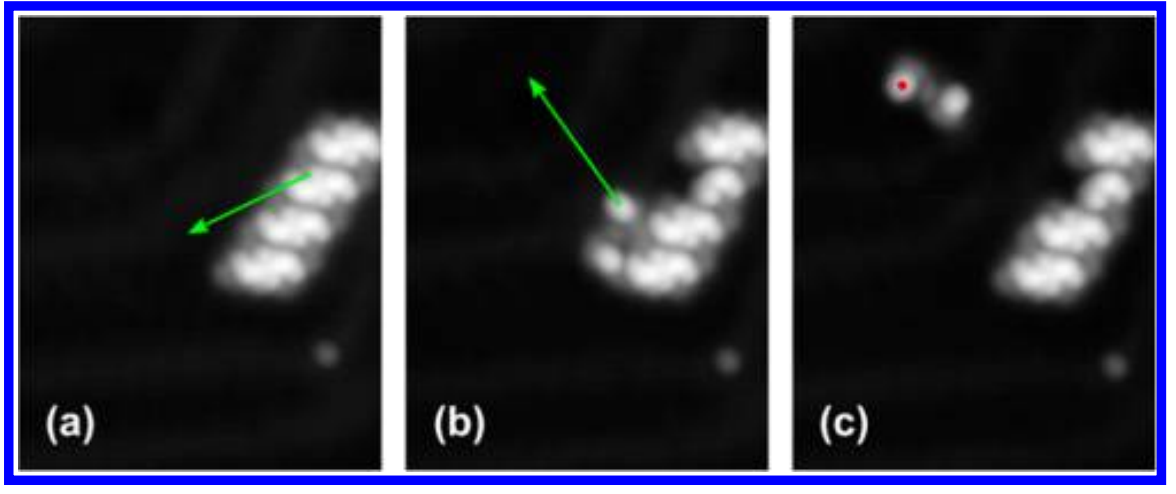

Figure 4. An example of the forcible molecule manipulation experiments using tunneling conditions less than 1/2 $R_{T}$ in Figure 3. Topographic images (a) before and $(b)$ after $R_{T}=120 \mathrm{M} \Omega$ manipulation. Using this condition, a structural transformation from syn to flat conformer occurs. (c) The so-produced flat conformer was manipulated with the STM tip away from its original four " $f$ " dimer line with $R_{T}=66$ MÎl' in order to isolate it to prevent the influence from other molecules during $d I / d V$ STM spectroscopic $m \Omega$. The specific tip trajectories during the manipulation to produce a flat conformer are indicated by the arrows in $(a)$ and $(b)$.

The tip location selected during the Figure 5 spectrum recording on this flat conformer is indicated by a dot in (c). Same STM image conditions as in Figure 2. Image size: $12 \mathrm{~nm} \times 15 \mathrm{~nm}$. 
mapping is of importance to confirm the flat conformation interpretation after the Figure 4 specific manipulation protocol. It is also very appropriate for determining the location of the maximum molecular orbital weight of its reduced and oxidized electronic states along the BBD molecular structure. Those maxima are known to be the principal port for intramolecular inelastic excitations induced by tunneling electrons in a way to bring energy to the molecular structure to move on a metallic surface with no mechanical push.

Figure 5a presents a typical $\mathrm{d} I / \mathrm{d} V$ spectrum recorded on a flat BBD molecule adsorbed on the $\mathrm{Au}(111)$ surface. Here, the STM tip apex was positioned at the center of one of the two lobes identified on the topographic image (see Figure 4c). After identifying the Au(111) surface states energy location $(\sim-0.5 \mathrm{~V})$ in this spectrum, two differential conductance peaks are observed, one at $-1.6 \mathrm{~V}$ and a small bump centered around $+2.7 \mathrm{~V}$. This gives an apparent electronic gap of about $4.3 \mathrm{eV}$ for a flat BBD molecule on the $\mathrm{Au}(111)$ surface to be compared to the $3.7 \mathrm{eV}$ (334 nm) UV optical gap observed for the syn and anti conformers in solution.

Performed exactly at these resonances, the Figure 5 very precise $\mathrm{d} I / \mathrm{d} V$ STM mapping permits to determine the spatial molecular orbital electronic distribution of the flat BBD reduced and oxidized electronic states at the origin of those two tunneling resonances. Monoelectronic constant current $\mathrm{d} I / \mathrm{d} V$ elastic scattering quantum chemistry (ESQC) $)^{34}$ image calculations were performed to compare with those experimental $\mathrm{d} I / \mathrm{d} V$ maps. The ESQC calculation is particularly welladapted to provide accurate STM images for large adsorbed molecules. ${ }^{35,36}$ These calculations confirm that the main contributor to the $-1.6 \mathrm{~V}$ resonance is the BBD highest occupied molecular orbital (HOMO) and the BBD lowest unoccupied molecular orbital (LUMO) to the $+2.7 \mathrm{~V}$ resonance. Those images were calculated starting from an optimized flat BBD conformation on the $\mathrm{Au}(111)$ surface. This confirms how the BBD molecule can be prepared in a flat conformation by STM single molecule manipulation. According to Figure 5 images, the central phenyl chassis is relatively electronically decoupled for the $\mathrm{Au}(111)$ surface.

\section{F. Step-by-Step Manipulation for Moving a flat BBD Conformer on the Au(111) Surface.}

To manipulate a BBD molecule by inelastic electron tunneling effects along a fcc portion of the $\mathrm{Au}(111)$ surface, the single molecule must capture enough energy from the tunneling current to pass over the fcc surface lateral diffusion barrier. One way to trigger the inelastic energy release on the $\mathrm{BBD}$ vibronic mode is to increase the tunneling current intensity through the molecule 


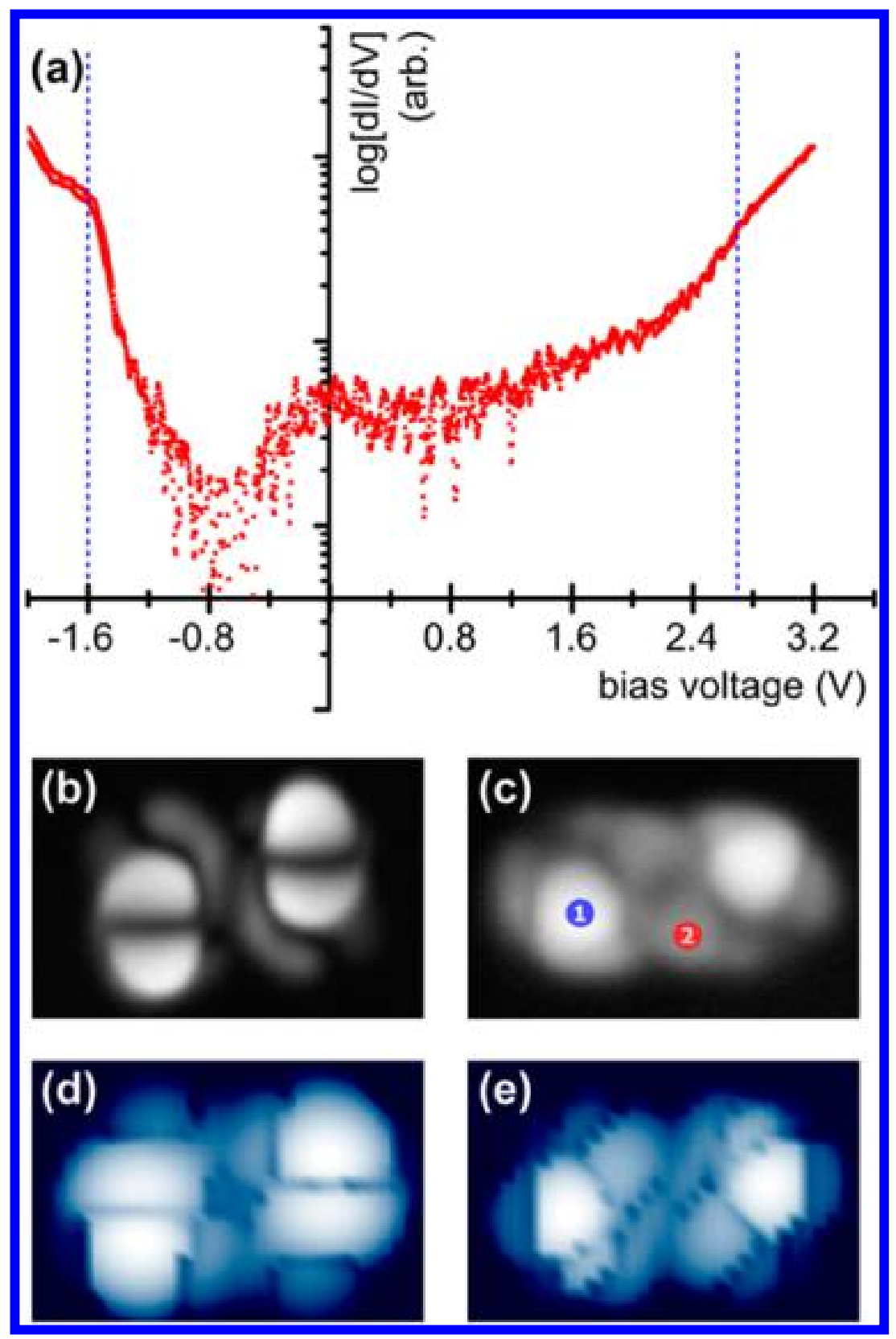

Figure 5. (a) The $d I / d V$ spectrum and $(b$ and $c) d I / d V$ maps recorded on flat BBD produced in Figure 4. The first tunneling resonances appear at $-1.6 \mathrm{~V}$ and $+2.7 \mathrm{~V}$ bias voltage (sample grounded on the LT-UHV 4-STM). A dI/dV map captures the spatial distribution of the electron density of the corresponding molecular electronic states contributing to the resonance. ${ }^{37}$ ( $d$ and e) At energies corresponding to HOMO and LUMO of the flat BBD monomer, monoelectronic ESQC STM calculated images are also presented for comparison. Resonance at -1.6 V appears to be mainly coming from the HOMO component of the imaged ground state and at $+2.7 \mathrm{~V}$ from the LUMO contribution of BBD reduced (image size: $3.6 \mathrm{~nm} \times 2.4 \mathrm{~nm}$ ). 
reaching its first low-lying reduced states. According to the $\mathrm{d} I / \mathrm{d} V$ spectrum in Figure $5 \mathrm{a}$, this can be achieved with a bias voltage applied to the tunnel junction greater than about $2 \mathrm{~V}$ to reach at least the tail of the $+2.7 \mathrm{~V} \mathrm{BBD}$ tunneling electronic resonance. Notice here that the energy captured by the vibronic modes of the BBD molecule will be a small fraction of the $2 \mathrm{eV}{ }^{38}$ To maximize the tunneling inelastic excitations, it is also usually taken for granted to position the STM tip apex at the locations at the highest electron density of the targeted in energy molecular electronic states. This strategy also helps to minimize the STM bias voltage range in a way not to destroy the molecule. ${ }^{11}$ The BBD $\mathrm{d} I / \mathrm{d} V$ images presented in Figure 5 are mapping those maxima and minima in its flat surface conformation. This mapping results from the electronic coupling between the tip apex and the molecular orbitals entering in the composition of resonating BBD electronic states when considering that those electronic states can be well described by a superposition of Slater determinants constructed using a molecular orbital basis set. ${ }^{37,39}$

The pixel-by-pixel construction of the Figure 5b,c maps results from the local measurement of the conductance of the BBD molecule at each pixel. In effect, this measurement projects the total BBD electronic probability density on a $2 \mathrm{D}$ plane. This is a very convenient way to identify where to position the tip for triggering a tunneling inelastic effect. For BBD, the highest electronic probability density (the highest $\mathrm{d} I / \mathrm{d} V$ ) sites are located on the BBD binaphthyl paddles as observed in Figure 5c.

As indicated in Figure 5c, when positioning the tip apex at location 1 on the BBD molecule and then ramping up the bias voltage further than $+2.3 \mathrm{~V}$ (but without reaching $+2.7 \mathrm{~V}$ ), the BBD changes its conformation with one naphthyl paddle going up the surface in a conformation similar to syn (syn/flat-like conformation, see Figure S21 in the Supporting Information). This conformational change occurs systematically opposite to the paddle been excited. On the corresponding reduced state potential energy surface, this indicates that the energy captured by the BBD molecule from the tunneling current is initiating a conformation change trajectory at the onset of the $+2.7 \mathrm{~V}$ resonance. Starting from the flat ground-state conformation, this trajectory certainly reaches a minimum on this reduced state potential energy manifold corresponding to the rotating up of a paddle to form a "syn/flat-like" conformation. Then, the BBD molecule relaxes in its ground state in this new stable "syn/flat-like" conformation not observable natively event after an STM mechanical lateral manipulation procedure. Notice that in the first approximation, the reduced state potential energy manifold can be explored using the potential energy surface of the BBD $\mathrm{S}_{1}$ vertically accessible excited electronic state. 
After having tried to induce the paddle vibrations directly, we have selected the electronic probability density maximum 2 as indicated in Figure 5c. For this new excitation location and as presented in left column of Figure 6 , when the bias voltage reaches about $+2.3 \mathrm{~V}$ and the tunneling current several hundred pico-amperes, the current intensity through the BBD suddenly jumps up due to the molecule one step lateral translation. This very reproducible behavior shows how a BBD molecule can be step-by-step driven by steps of $0.29 \mathrm{~nm}$ on an fcc flat area on the Au(111) surface. To be more precise on the inelastic manipulation direction, an atomic resolved image of the $\mathrm{Au}(111)$ surface recorded using molecule terminated tip is inserted in Figure 6a to certify the accurate moving direction: exactly one of $\langle 11 \overline{0}\rangle$ orientations of the $\mathrm{Au}(111)$ surface inits fcc portion. The interatomic gold atom distance along those orientations is $0.288 \mathrm{~nm}$, in complete agreement with the experimentally observed $0.29 \mathrm{~nm}$ long step motion per voltage ramp. As presented in Figure 6, a controllable inelastic motion is only possible when the molecule lies parallel to and in between two herringbones of the reconstruction. When the molecule sits on a herringbone (even when only one paddle end is laying on it), it is stuck and difficult to manipulate inelastically, as also recently observed with a windmill molecule. ${ }^{40}$

For a BBD molecule laying parallel to the herringbones and located at the fcc portion of the $\mathrm{Au}(111)$ surface, the probability of a controllable stepwise motion is about $50 \%$ after a single shot bias voltage ramp. Incidentally, the probability of molecule motion regardless the adsorption site is around 5\% and the probability of a breaking or a conformation changes is around $3 \%$. As a consequence and after a three consecutive lateral step-by-step motions, the BBD molecule is stopped because of herringbone lateral diffusion barrier and must be reprepared for a new run like with the windmill molecule. ${ }^{40}$ Here, the BBD molecule must be manipulated with care not to open a conformation change path on its reduced state potential energy surface nor a chemical reaction path breaking some of its chemical bonds leading to the final destruction of the molecule since a molecule is often very unstable under high positive STM bias voltage pulses. ${ }^{7,41}$

While exciting the BBD molecule at two different spatial locations of the same resonance maxima, the difference of mechanical response is a nice indication of how the electronic coupling between the tip apex and the electronic states of a molecule can give rise to different mechanical responses. Here and during an STM excitation (or imaging), the effective lateral extension of the tunneling electrons inelastic excitation is much narrower than the BBD molecular orbitals spatial lateral extension. As a consequence, the electronic coupling between the tip apex and the BBD molecule is very local. For each tip positioning on the molecule, this brings out a very specific 


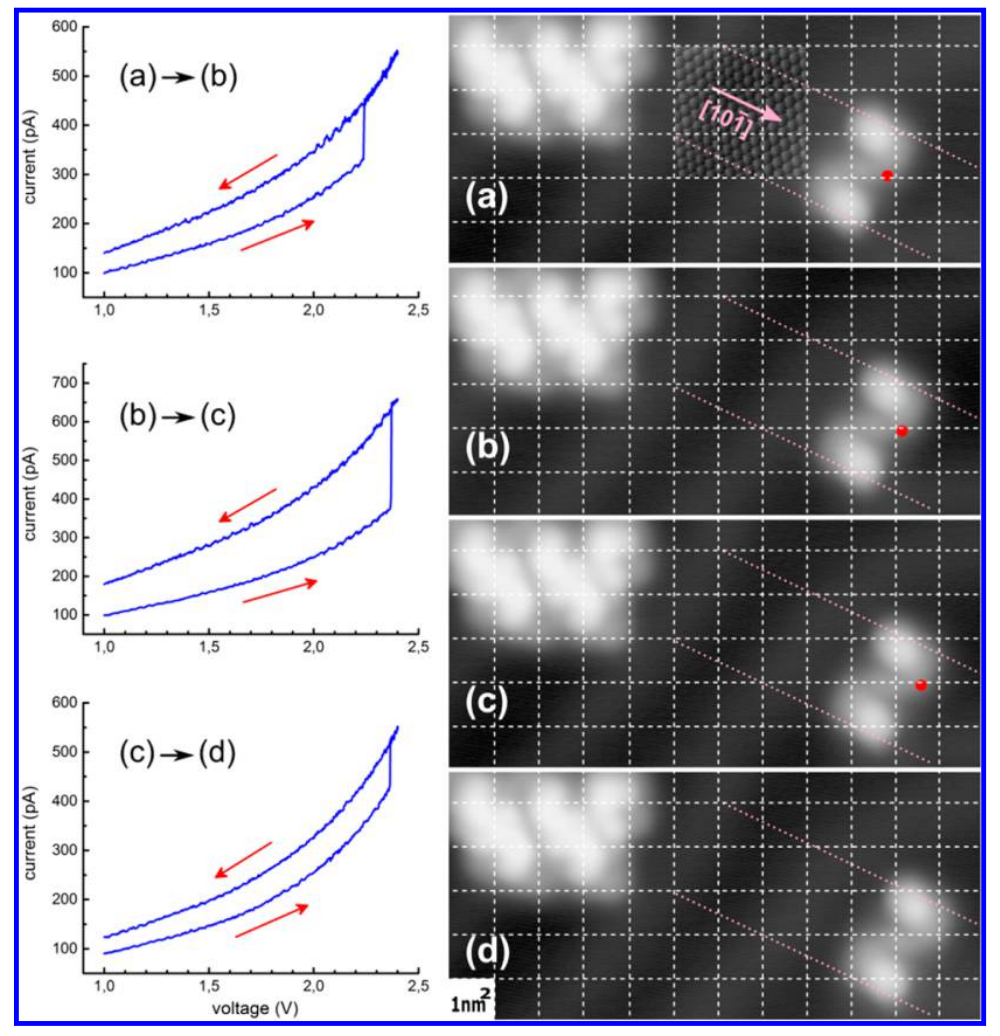

Figure 6. Manipulation of a BBD molecule on the Au(111) surface using inelastic electrons tunneling excitations. $(a-d)$ A series of images demonstrating the BBD molecule motion along

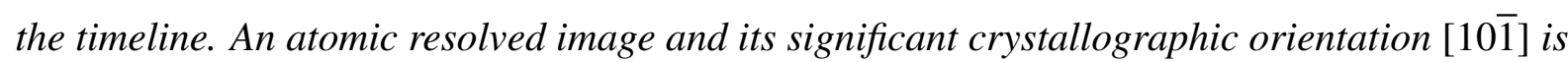
also presented. The red dots on each image indicate the tip apex location for applying the bias voltage ramp, resulting in the measured tunneling current. The moving direction, of a BBD

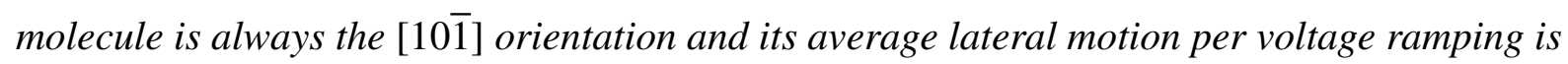
around $0.29 \mathrm{~nm}$, which perfectly matches with the $0.288 \mathrm{~nm}$ interatomic distance on $\mathrm{Au}(111)$ in the $\langle\overline{1} \overline{10}\rangle$ surface directions. The voltage ramp duration from +1.0 to $+2.3 \mathrm{~V}$ was $20 \mathrm{~s}$ in each case. Left column shows I - V characteristics for each inelastic event. Right column: $12.0 \mathrm{~nm} \times$ $5.6 \mathrm{~nm}$ constant current STM images recorded at $V=+0.5 \mathrm{~V}$ and $\mathrm{I}=10 \mathrm{pA}$. In each image, top left, two dimers and one BBD monomer in a syn conformation have been imaged at the same time to provide a clear measurement of the BBD molecule motion per excitation. 
superposition of BBD molecular orbitals to contribute to the motion as also observed for STM

imaging in the case, for example, of an hexabenzocoronene (HBC) molecule. ${ }^{42}$ This can trigger a large conformation of the paddle for one tip apex location or a gentle stepwise lateral motion for another location supposing that the effective potential energy surface built up from this superposition is different in the two cases.

For negative applied bias voltage, we have also tried the same strategy by locating the tip apex at one of the many maxima indicated in Figure 5b. No movement of the BBD molecule was observed down to a bias voltage ramp reaching a maximum of $-2.0 \mathrm{~V}$ with several $\mathrm{nA}$ of tunneling current intensity. We do not yet have a detailed explanation of this observation.

\section{CONCLUSION}

A bisbinaphthyldurene (BBD) molecule was designed, synthesized, and deposited on an Au(111) surface, mechanically manipulated with a STM tip, and then with the STM inelastic contribution of the tunneling current passing through this molecule. The BBD molecule is equipped with two lateral binaphthyl paddles mounted on a very simple central phenyl chassis to separate it from the supporting surface. Single molecule STM lateral mechanical manipulation must be first performed for this molecule to reach a flat conformation on the $\mathrm{Au}(111)$ surface since its native surface conformation on $\mathrm{Au}(111)$ is a perpendicular dimer conformation. Once a BBD molecule was prepared in its flat conformation, $\mathrm{d} I / \mathrm{d} V$ molecular orbital mapping was performed to determine the best tip apex location to free up this molecule inelastically for a manipulation on an $\mathrm{Au}(111) \mathrm{fcc}$ flat terrace. The intuitive on-paddle excitation is not a good entry port for an inelastic tunnel manipulation since it leads to a drastic conformation change of the BBD molecule entering in competition with its step-by-step motion on the $\mathrm{Au}(111)$ surface. We have demonstrated that on the molecule and nearby the paddle location, there exists another energy entry port where the BBD molecule remains flat on the surface and can be laterally manipulated step-by-step with a step of about $0.29 \mathrm{~nm}$ per excitation. The BBD molecule was used by the MANA-NIMS Japanese team during the first international nanocar race in Toulouse. ${ }^{43}$ 


\section{MATERIALS AND METHODS}

The synthetic procedure of BBD is described in the Supporting Information. The STM experiments were conducted as follows. The BBD molecules were deposited on a Au(111) single crystal surface previously cleaned by standard metal surface UHV preparation methods consisting of several cycles of ion sputtering and subsequent annealing. ${ }^{6,10}$ The BBD molecules were sublimated from about $3 \mathrm{mg}$ of the colorless BBD molecular powder by heating a Kentax quartz crucible at $563 \mathrm{~K}$ during $30 \mathrm{~s}$. The gold substrate temperature was kept below $323 \mathrm{~K}$ during this deposition. The evaporation parameters were selected in a way to deposit a minute amount of BBD molecules to produce a submonolayer coverage in order to leave enough large molecule-free areas on the clean $\mathrm{Au}(111)$ surface to be able to use the STM single molecule manipulation protocol. The Au(111) sample was then loaded on the STM sample stage kept at cryogenic temperature (LT) and rapidly cooled down to $\sim 5 \mathrm{~K}$. All low-temperature ultrahigh-vacuum scanning tunneling microscopy (LT-UHV STM) experiments presented, that is, constant current imaging, molecule manipulations, tunneling spectroscopic measurements, and intramolecular $\mathrm{d} I / \mathrm{d} V$ mapping were performed on one of the four STM heads of our new ScientaOmicron LT-UHV 4 independent STM instrument. ${ }^{44}$

* E-mail: we-hyo.soe@ cemes.fr

$\dagger$ Present Address: Department of Chemical Engineering, Kyushu University, 744 Motooka, Nishi-ku, Fukuoka 819-0395, Japan.

† E-mail: Nakanishi.Waka@nims.go.jp.

1 Eigler, D. M.; Schweizer, E. K. Positioning Single Atoms with a Scanning Tunnelling Microscope. Nature $1990,344,524-526$.

2 Bartels, L.; Meyer, G.; Rieder, K.-H. Basic Steps of Lateral Manipulation of Single Atoms and Diatomic Clusters with a Scanning Tunneling Microscope Tip. Phys. Rev. Lett. 1997, 79, 697-700.

3 Bouju, X.; Joachim, C.; Girard, C.; Tang, H. Mechanics of $(\mathrm{Xe})_{N}$ Atomic Chains under STM Manipulation. Phys. Rev. B: Condens. Matter Mater. Phys. 2001, 63, 085415.

4 Jung, T. A.; Schlittler, R. R.; Gimzewski, J. K.; Tang, H.; Joachim, C. Controlled Room-Temperature Positioning of Individual Molecules: Molecular Flexure and Motion. Science 1996, 271, 181-184. 
5 Grill, L.; Rieder, K.-H.; Moresco, F.; Rapenne, G.; Stojkovic, S.; Bouju, X.; Joachim, C. Rolling a Single Molecular Wheel at the Atomic Scale. Nat. Nanotechnol. 2007, 2, 95-98.

6 Manzano, C.; Soe, W.-H.; Wong, H. S.; Ample, F.; Gourdon, A.; Chandrasekhar, N.; Joachim, C. Stepby-Step Rotation of a Molecule-Gear Mounted on an Atomic-Scale Axis. Nat. Mater. 2009, 8, 576-579.

7 Nickel, A.; Ohmann, R.; Meyer, J.; Grisolia, M.; Joachim, C.; Moresco, F.; Cuniberti, G. Moving Nanostructures: Pulse-Induced Positioning of Supramolecular Assemblies. ACS Nano 2013, 7, 191-197.

${ }^{8}$ Lastapis, M.; Martin, M.; Riedel, D.; Hellner, L.; Comtet, G.; Dujardin, G. Picometer-Scale Electronic Control of Molecular Dynamics Inside a Single Molecule. Science 2005, 308, 1000-1003.

9 Hla, S.-W. Scanning Tunneling Microscope Atom and Molecule Manipulations: Realizing Molecular Switches and Devices. Jpn. J. Appl. Phys. 2008, 47, 6063-6069.

10 Stipe, B. C.; Rezaei, M. A.; Ho, W. Inducing and Viewing the Rotational Motion of a Single Molecule. Science 1998, 279, 1907-1909.

11 Dujardin, G.; Walkup, R. E.; Avouris, Ph. Dissociation of Individual Molecules with Electrons from the Tip of a Scanning Tunneling Microscope. Science 1992, 255, 1232-1235.

12 Langlais, V.; Schlittler, R. R.; Tang, H.; Gourdon, A.; Joachim, C.; Gimzewski, J. K. Spatially Resolved Tunneling Along a Molecular Wire. Phys. Rev. Lett. 1999, 83, 2809-2812.

13 Joachim, C.; Tang, H.; Moresco, F.; Rapenne, G.; Meyer, G. The Design of a Nanoscale Molecular Barrow. Nanotechnology 2002, 13, 330-335.

14 Shirai, Y.; Osgood, A. J.; Zhao, Y.; Kelly, K. F.; Tour, J. M. Directional Control in Thermally Driven Single-Molecule Nanocars. Nano Lett. 2005, 5, 2330-2334.

15 Koumura, N.; Zijlstra, R. W. J.; van Delden, R. A.; Harada, N.; Feringa, B. L. Light-Driven Monodirectional Molecular Rotor. Nature 1999, 401, 152-155.

16 Morin, J. F.; Shirai, Y.; Tour, J. M. En Route to a Motorized Nanocar. Org. Lett. 2006, 8, 1713-1716.

17 Chiang, P. T.; Mielke, J.; Godoy, J.; Guerrero, J. M.; Alemany, L. B.; Villagómez, C.J.; Saywell, A.; Grill,L.; Tour, J.M. Toward a Light-Driven Motorized Nanocar: Synthesis and Initial Imaging of Single Molecules. ACS Nano 2012, 6, 592-597.

18 Kudernac, T.; Ruangsupapichat, N.; Parschau, M.; Maciá, B.; Katsonis, N.; Harutyunyan, S. R.; Ernst, K.-H.; Feringa, B. L. Electrically Driven Directional Motion of a Four-Wheeled Molecule on a Metal Surface. Nature 2011, 479, 208-211.

19 Launay, J.-P. Single Molecules to Practical Devices. In From Non-Covalent Assemblies to Molecular Machines; Sauvage, J.-P., Gaspard, P., Eds.; Wiley-VCH: Weinheim, 2010; pp 381-428. 
20 Alemani, M.; Selvanathan, S.; Ample, F.; Peters, M. V.; Rieder, K. H.; Moresco, F.; Joachim, C.; Hecht, S.; Grill, L. Adsorption and Switching Properties of Azobenzene Derivatives on Different Noble Metal Surfaces: $\mathrm{Au}(111), \mathrm{Cu}(111)$, and $\mathrm{Au}(100)$. J. Phys. Chem. C 2008, 112, 10509-10514.

21 Sainoo, Y.; Kim, Y.; Komeda, T.; Kawai, M.; Shigekawa, H. Observation of Cis-2-Butene Molecule on Pd(110) by Cryogenic STM: Site Determination Using Tunneling-Current-Induced Rotation. Surf. Sci. 2003, 536, L403-L407.

22 Henzl, J.; Morgenstern, K. An Electron Induced Two-Dimensional Switch Made of Azobenzene Derivatives Anchored in Supramolecular Assemblies. Phys. Chem. Chem. Phys. 2010, 12, 6035-6044.

23 Valeur, B.; Berberan-Santos, M. N. Structural Effects on Fluorescence Emission. In Molecular Fluorescence: Principles and Applications; Wiley-VCH: Weinheim, 2012; pp 87-92.

24 Setnička, V.; Urbanová, M.; Bouř, P.; Král, V.; Volka, K. Vibrational Circular Dichroism of 1,1'Binaphthyl Derivatives: Experimental and Theoretical Study. J. Phys. Chem. A 2001, 105, 8931-8938.

25 Di Bari, L.; Pescitelli, G.; Salvaori, P. Conformational Study of 2,2'-Homosubstituted 1,1'-Binaphthyls by Means of UV and CD Spectroscopy. J. Am. Chem. Soc. 1999, 121, 7998-8004.

26 Fujiyoshi, S.; Takeuchi, S.; Tahara, T. Time-Resolved Impulsive Stimulated Raman Studies of 1,1'Binaphthyl in the Excited State: Low-Frequency Vibrations and Conformational Relaxation. J. Phys. Chem. A 2004, 108, 5938-5943.

27 Hochstrasser, R. M. The Effect of Intramolecular Twisting on the Emission Spectra of Hindered Aromatic Molecules. Can. J. Chem. 1961, 39, 459-470.

28 Pu, L. 1,1'-Binaphthyl Dimers, Oligomers, and Polymers: Molecular Recognition, Asymmetric Catalysis, and New Materials. Chem. Rev. 1998, 98, 2405-2494.

29 Nishizaka, M.; Mori, T.; Inoue, Y. Experimental and Theoretical Studies on the Chiroptical Properties of Donor-Acceptor Binaphthyls. Effects of Dynamic Conformer Population on Circular Dichroism. J. Phys. Chem. Lett. 2010, 1, 1809-1812.

30 Meca, L.; Řeha, D.; Havlas, Z. Racemization Barriers of 1,1'- Binaphthyl and 1,1'-Binaphthalene-2,2'Diol: A DFT Study. J. Org. Chem. 2003, 68, 5677-5680.

31 Ishikawa, D.; Mori, T.; Yonamine, Y.; Nakanishi, W.; Cheung, D. L.; Hill, J. P.; Ariga, K. Mechanochemical Tuning of the Binaphthyl Conformation at the Air-Water Interface. Angew. Chem., Int. Ed. 2015, 54, 8988-8991.

32 Mori, T.; Ishikawa, D.; Yonamine, Y.; Fujii, Y.; Hill, J. P.; Ichinose, I.; Ariga, K.; Nakanishi, W. Mechanically Induced Opening-Closing Action of Binaphthyl Molecular Pliers: Digital Phase Transition 
versus Continuous Conformational Change. ChemPhysChem 2017, 18, 1470-1474.

33 Friedel, R. A.; Orchin, M.; Reggel, L. Steric Hindrance and Short Wave Length Bands in the Ultraviolet Spectra of Some Naphthalene and Diphenyl Derivatives. J. Am. Chem. Soc. 1948, 70, 199-204.

34 Sautet, P.; Joachim, C. Calculation of the Benzene on Rhodium STM Images. Chem. Phys. Lett. 1991, 185, 23-30.

35 Yu, M.; Xu, W.; Kalashnyk, N.; Benjalal, Y.; Nagarajan, S.; Masini, F.; Lægsgaard, E.; Hliwa, M.; Bouju, X.; Gourdon, A.; Joachim, C.; Besenbacher, F.; Linderoth, T. R. From Zero to Two Dimensions: Supramolecular Nanostructures Formed from Perylene-3,4,9,10-Tetracarboxylic Diimide (PTCDI) and $\mathrm{Ni}$ on the $\mathrm{Au}(111)$ Surface through the Interplay between Hydrogen-Bonding and Electrostatic MetalOrganic Interactions. Nano Res. 2012, 5, 903-916.

36 Saywell, A.; Greń, W.; Franc, G.; Gourdon, A.; Bouju, X.; Grill, L. Manipulating the Conformation of Single Organometallic Chains on Au(111). J. Phys. Chem. C 2014, 118, 1719-1728.

37 Soe, W.-H.; Manzano, C.; De Sarkar, A.; Chandrasekhar, N.; Joachim, C. Direct Observation of Molecular Orbitals of Pentacene Physisorbed on Au(111) by Scanning Tunneling Microscope. Phys. Rev. Lett. $2009,102,176102$.

38 Ohmann, R.; Meyer, J.; Nickel, A.; Echevarria, J.; Grisolia, M.; Joachim, C.; Moresco, F.; Cuniberti, G. Supramolecular Rotor and Translator at Work: On-Surface Movement of Single Atoms. ACS Nano 2015, 9, 8394-8400.

39 Krüger, T.; Eisenhut, F.; Alonso, J. M.; Lehmann, T.; Guitián, E.; Pérez, D.; Skidin, D.; Gamaleja, F.; Ryndyk, D. A.; Joachim, C.; Peña, D.; Moresco, F.; Cuniberti, G. Imaging the Electronic Structure of On-Surface Generated Hexacene. Chem. Commun. 2017, 53, 1583-1586.

40 Eisenhut, F.; Durand, C.; Moresco, F.; Launay, J. P.; Joachim, C. Training for the 1st International Nanocar Race: The Dresden Molecule-Vehicle. Eur. Phys. J.: Appl. Phys. 2016, 76, 10001.

41 Manzano, C.; Soe, W.-H.; Joachim, C. High Voltage STM Imaging of Single Copper Phthalocyanine. In Imaging and manipulating Molecular Orbitals. Advances in Atom and Single Molecule Machines; Grill, L., Joachim, C., Eds.; Springer: Berlin, 2013; p 15.

42 Soe, W.-H.; Wong, H. S.; Manzano, C.; Grisolia, M.; Hliwa, M.; Feng, X.; Müllen, K.; Joachim, C. Mapping the Excited States of Single Hexa-Peri-Benzocoronene Oligomers. ACS Nano 2012, 6, 32303235 .

43 Joachim, C.; Rapenne, G. Molecule Concept Nanocars: Chassis, Wheels, and Motors? ACS Nano 2013, 7, 11-14. 
44 Yang, J.; Sordes, D.; Kolmer, M.; Martrou, D.; Joachim, C. Imaging, Single Atom Contact and Single Atom Manipulations at Low Temperature Using the New ScientaOmicron LT-UHV-4 STM. Eur. Phys. J.: Appl. Phys. 2016, 73, 10702.

\section{ACKNOWLEDGMENTS}

We thank Dr. Y. Okawa, Dr. T. Uchihashi, and Dr. K. Sagisaka in NIMS for prescreening of STM conditions and helpful discussions and Prof. M. Aono for its continuous support during this work.

Funding WPI MANA MEXT program and by JSPS KAKENHI (16H07436, JP16H06518, 26790003). M.K. acknowledges financial support received from the Foundation for Polish Science (FNP). We thank TOYOTA as an official sponsor for our NIMS MANA team in the nanocar race. ${ }^{43}$

Notes The authors declare no competing financial interest. 


\section{SUPPORTING INFORMATION}

\section{1- General}

Analytical thin-layer chromatography (TLC) was performed on a glass plate coated with silica gel (230-400 mesh, $0.25 \mathrm{~mm}$ thickness) containing a fluorescent indicator (silica gel 60F254, Merck). Flash silica gel column chromatography was performed on silica gel 60N (spherical and neutral gel, 40-50 $\mu \mathrm{m}$, Kanto). Infrared (IR) spectra were recorded on Thermo Scientific Nicolet NEXUS 670 FT-IR and were reported as wavenumbers $(v)$ in $\mathrm{cm}^{-1}$. Proton $\left({ }^{1} \mathrm{H}\right)$ and carbon $\left({ }^{13} \mathrm{C}\right)$ nuclear magnetic resonance (NMR) spectra were recorded on a JEOL JNM-ECA400 spectrometer. Mass spectra were obtained on an Applied Biosystems Voyager DE STR SI-3 instrument (MALDITOF MS). UV-Vis absorption spectra were obtained on JASCO V-670. Circular dichroism (CD) spectra were obtained on JASCO, J-820.

\section{Materials}

Solvents and materials were purchased from Aldrich, Tokyo Kasei Chemical Co. or Wako Chemical Co., and were used without further purification.

\section{Synthesis}

To a mixture of $(R)-(+)-1,1$ '-bi(2-naphthol) (1.00 g, $3.49 \mathrm{mmol})$ and $\mathrm{CsCO}_{3}(2.84 \mathrm{~g}, 8.73 \mathrm{mmol})$ in dry acetone $(100 \mathrm{~mL})$ was added 1,2,4,5-tetrakis(bromomethyl)benzene (715 mg, $1.59 \mathrm{mmol}$ ) and the mixture was refluxed $48 \mathrm{~h}$. The mixture was extracted with $\mathrm{CH}_{2} \mathrm{Cl}_{2}(2 \times 200 \mathrm{~mL})$ and concentrated in vacuo. The crude material was purified by silica gel column chromatography (eluent: $\mathrm{CH}_{2} \mathrm{Cl}_{2} / \mathrm{Hexane}$ ) to give pure desired compound, bisbinaphthyldurene (BBD) (410 mg, 37\%). The compound was further purified by sublimation $\left(<300{ }^{\circ} \mathrm{C}\right)$ for the STM experiments. Mp $>250^{\circ} \mathrm{C}$; FT-IR $\left(\mathrm{KBr}, \mathrm{cm}^{1}\right)$ 3047, 2934, 2880, 1918, 1593, 1472, 1321, 1244, 1147, 1079, 1009, 893, 805, 749; ${ }^{1} \mathrm{H}$ NMR (400 MHz, $\left.\mathrm{CDCl}_{3}\right) \delta 5.15(\mathrm{~d}, J=11.2 \mathrm{~Hz}, 4 \mathrm{H}), 5.21(\mathrm{~d}, J=11.2 \mathrm{~Hz}$, 4H), 7.16 (ddd, $J=7.6,7.6,0.8 \mathrm{~Hz}, 4 \mathrm{H}), 7.18$ (dd, $J=7.6,7.2 \mathrm{~Hz}, 4 \mathrm{H}), 7.27$ (ddd, $J=7.2,7.2$, $1.4 \mathrm{~Hz}, 4 \mathrm{H}), 7.32(\mathrm{~s}, 2 \mathrm{H}), 7.46(\mathrm{~d}, J=9.0 \mathrm{~Hz}, 4 \mathrm{H}), 7.75(\mathrm{~d}, J=7.6 \mathrm{~Hz}, 4 \mathrm{H}), 7.80(\mathrm{~d}, J=9.0 \mathrm{~Hz}$,

$4 \mathrm{H}) ;{ }^{13} \mathrm{C}$ NMR (400 MHz, $\left.\mathrm{CDCl}_{3}\right): \delta 71.4,117.0,121.6,123.9,126.1,126.3,128.1,129.3,129.8$, 133.4, 134.5, 136.6, 154.4; HRMS (MALDI, 9- nitroanthracene) $\mathrm{m} / z$ calcd for $\mathrm{C}_{50} \mathrm{H}_{34} \mathrm{O}_{4}[\mathrm{M}]^{+}$ 


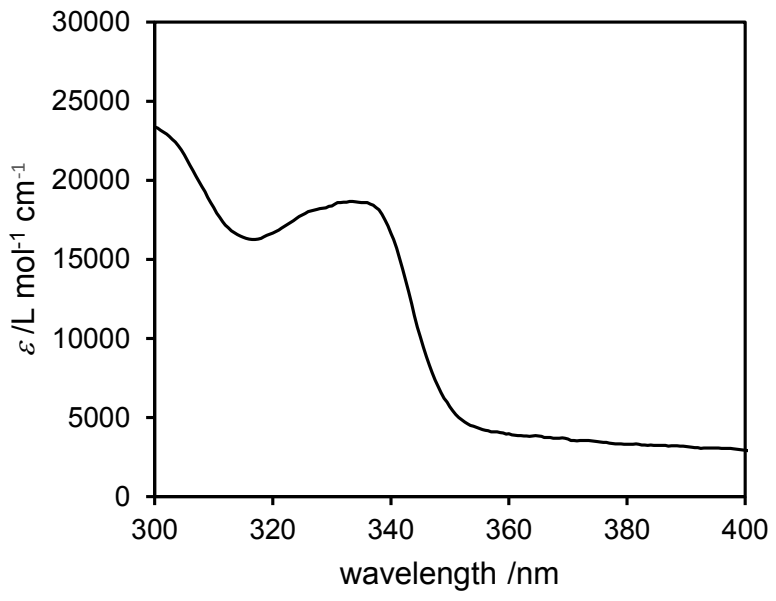

Figure S1. UV-vis absorption spectra of BBD in THF $\left(5.6 \times 10^{-6} \mathrm{M}\right)$.

698.2452, found 698.2451; $\mathrm{C}_{100} \mathrm{H}_{68} \mathrm{O}_{8}[\mathrm{M}]^{+}$1396.4909, found 1396.4905.

\section{Photophysical properties}

See Fig. S1 and Fig. S2.

\section{NMR spectra}

See Fig. S3 to Fig. S15.

\section{Theoretical calculations (DFT)}

All calculations were performed using the Gaussian 09 program, ${ }^{1}$ and the results were analyzed and visualized on GaussView 5.0.9. Calculations were performed at the density functional theory 


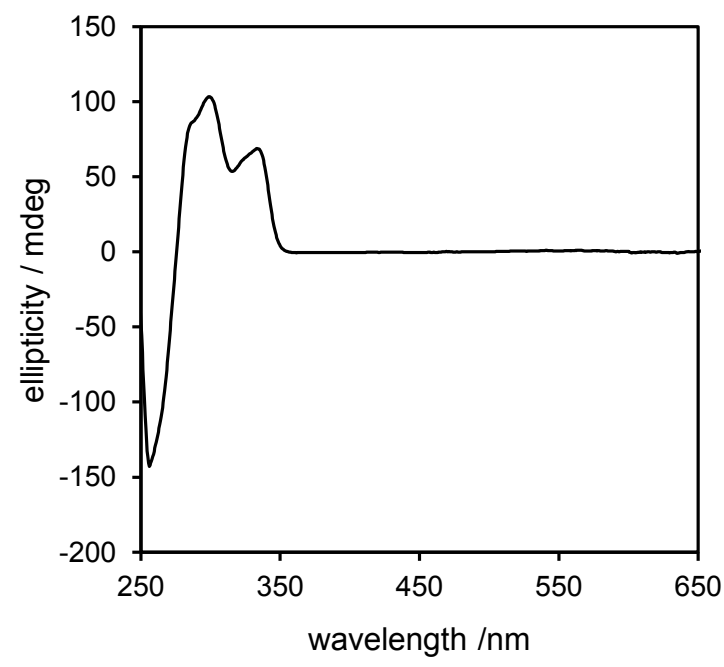

Figure S2. $C D$ spectra of $B B D$ in $T H F\left(1.0 \times 10^{-5} M\right)$.

(DFT) level with the B3LYP functional, the gradient correction of the exchange functional by Becke $^{2,3}$ and the correlation functional by Lee, Yang and Parr, ${ }^{4}$ and the $6-31 \mathrm{G}(\mathrm{d}, \mathrm{p})$ split valence plus polarization basis set ${ }^{5-8}$ was used. Relaxed $S_{1}$ structures (= optimized structures in $S_{1}$ state) were calculated by time-dependent (TD) DFT.

See Tab. S1, Tab. S2, Fig. S16, Fig. S17 


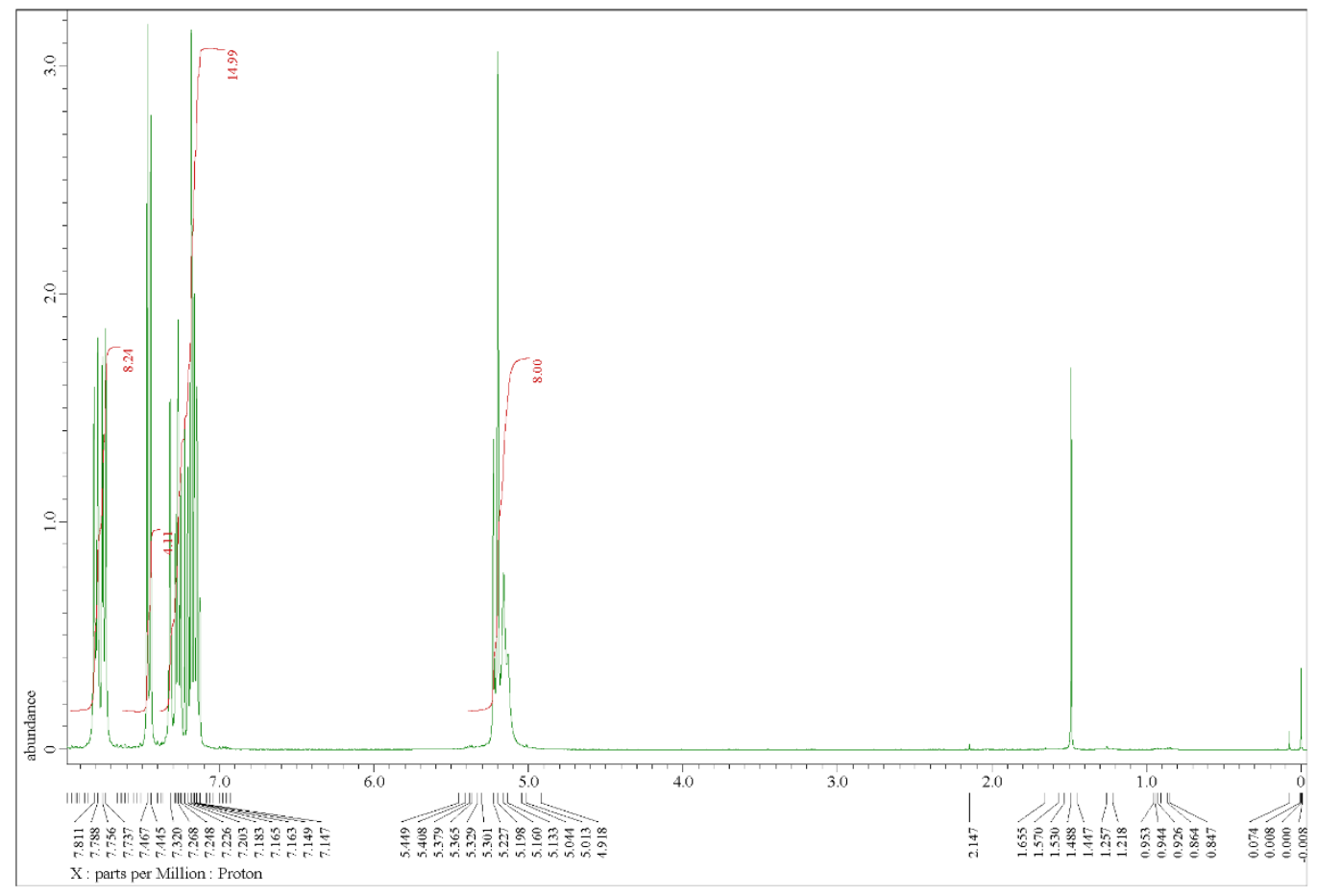

Figure S3. ${ }^{1} H$ NMR of $B B D$. 


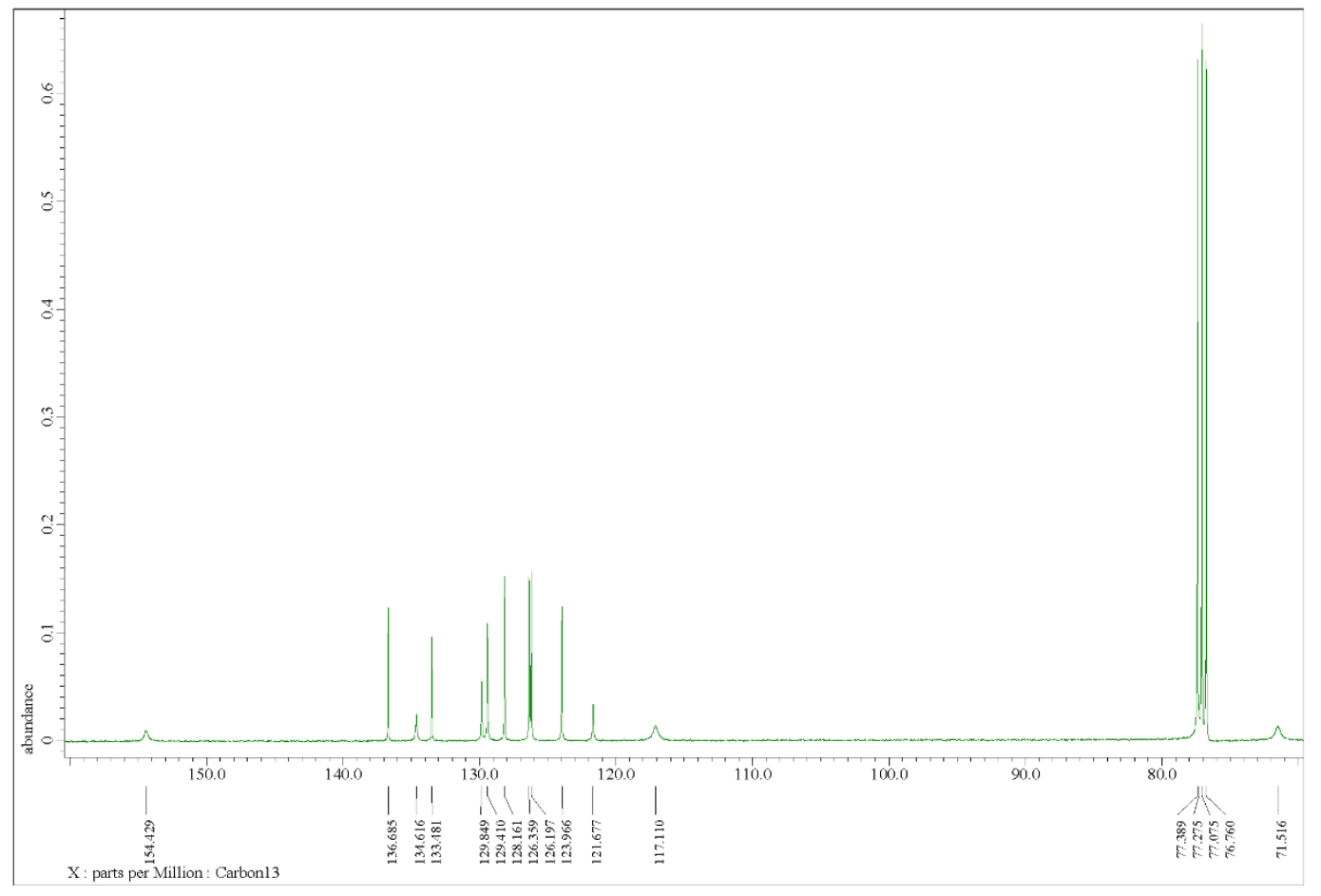

Figure S4. ${ }^{13} C N M R$ of $B B D$. 


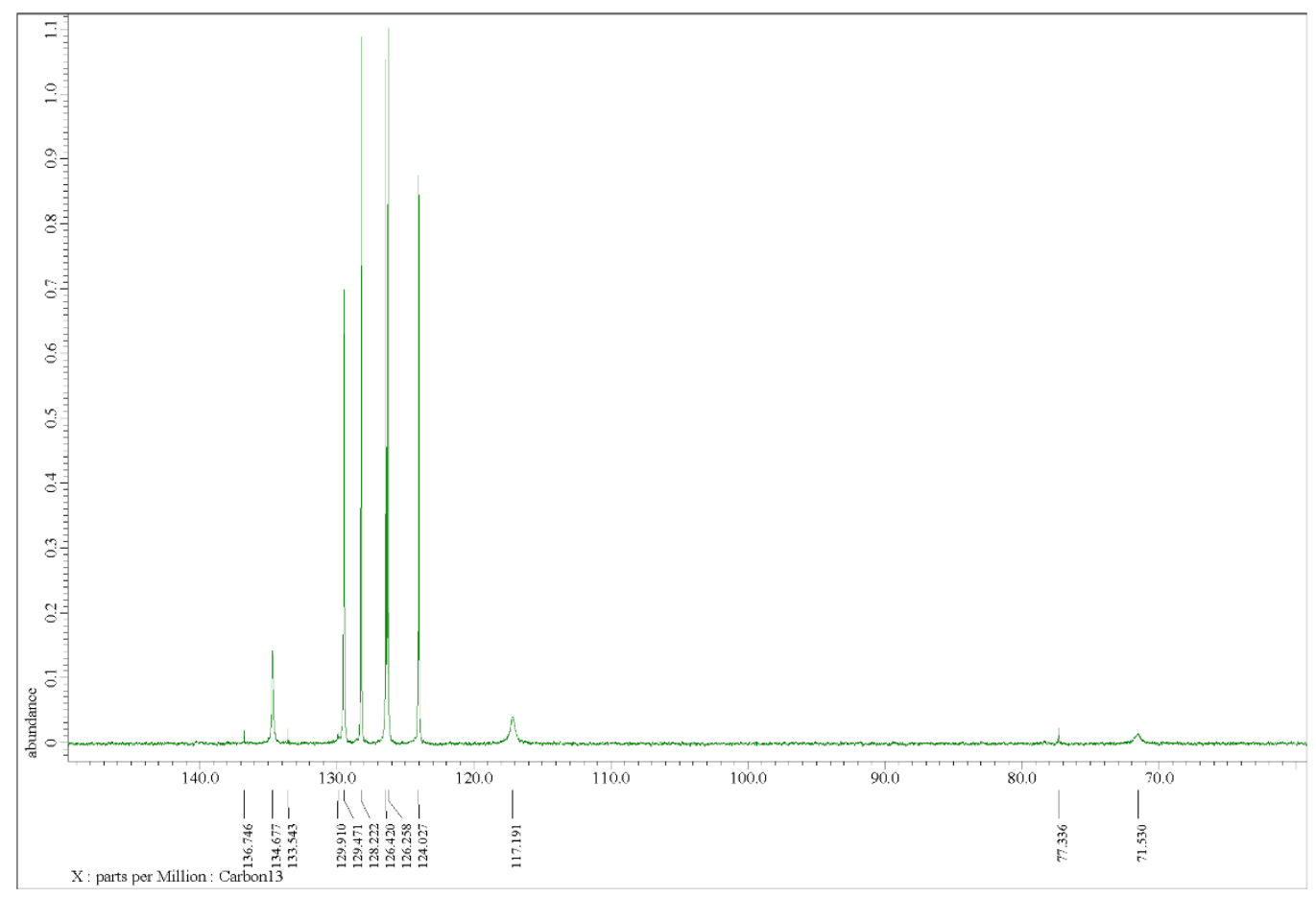

Figure S5. DEPT 90 of $B B D$. 


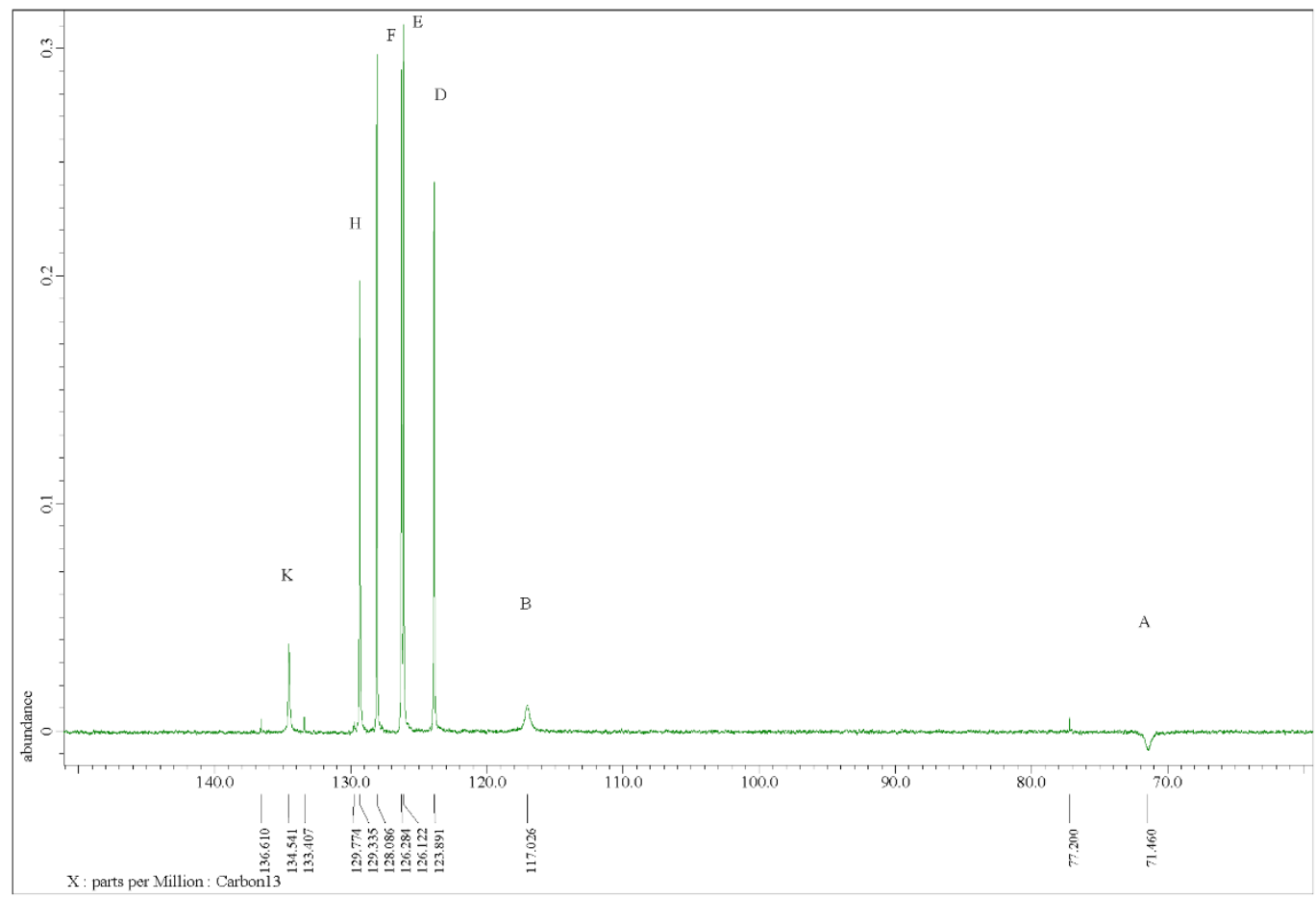

Figure S6. $D E P T 135$ of $B B D$. 


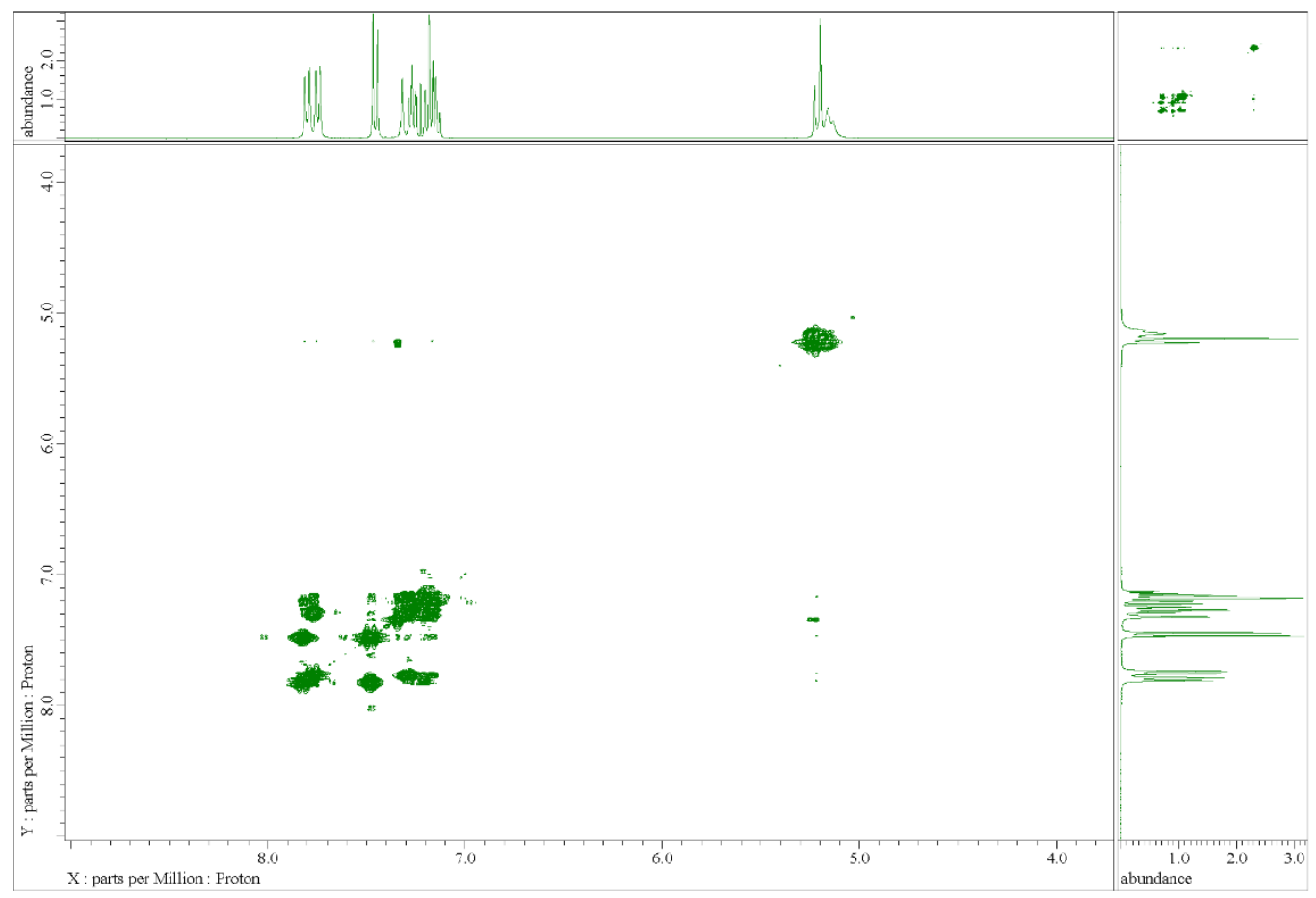

Figure S7. $C O S Y$ of $B B D$. 


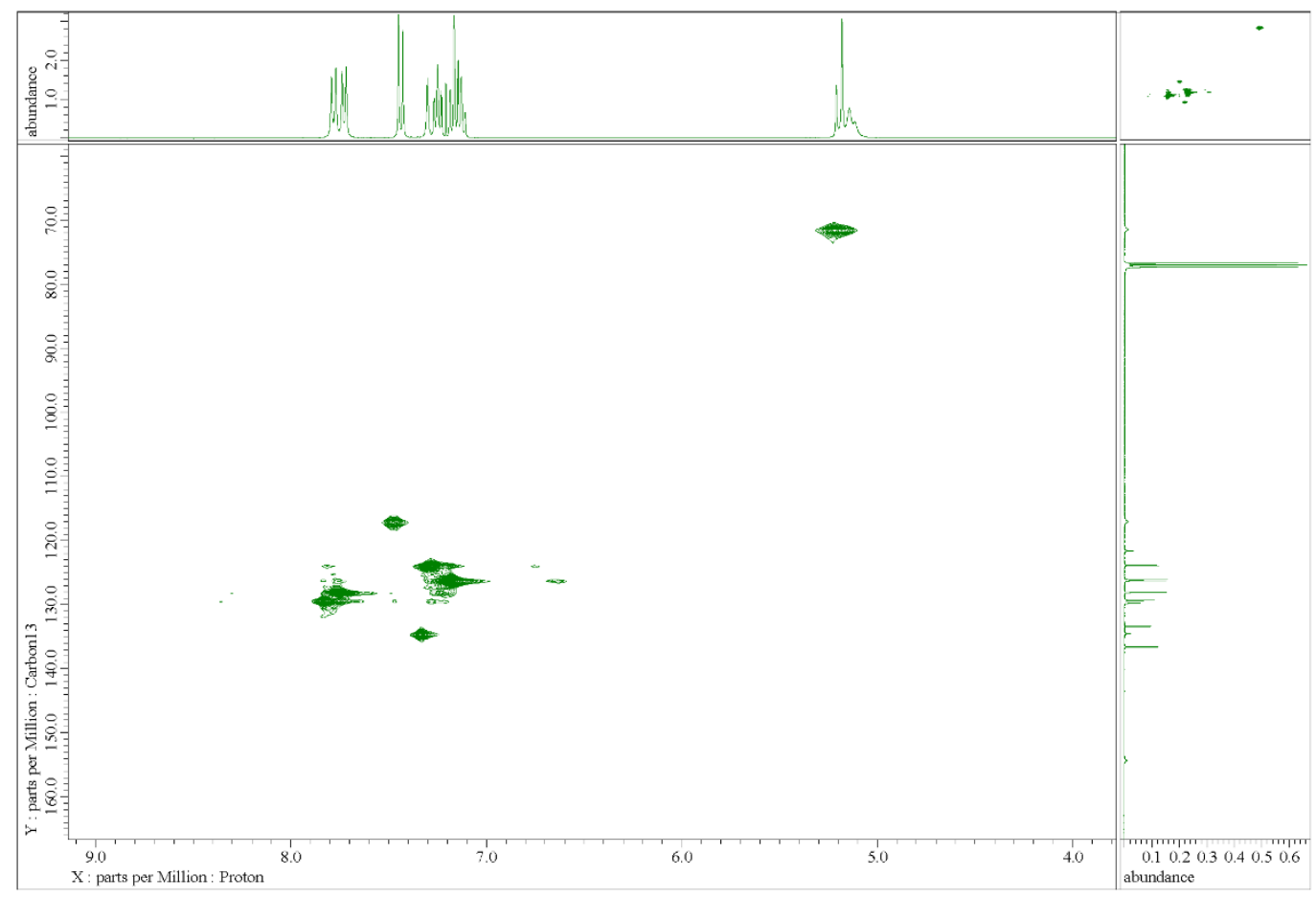

Figure S8. $H M Q C$ of $B B D$. 


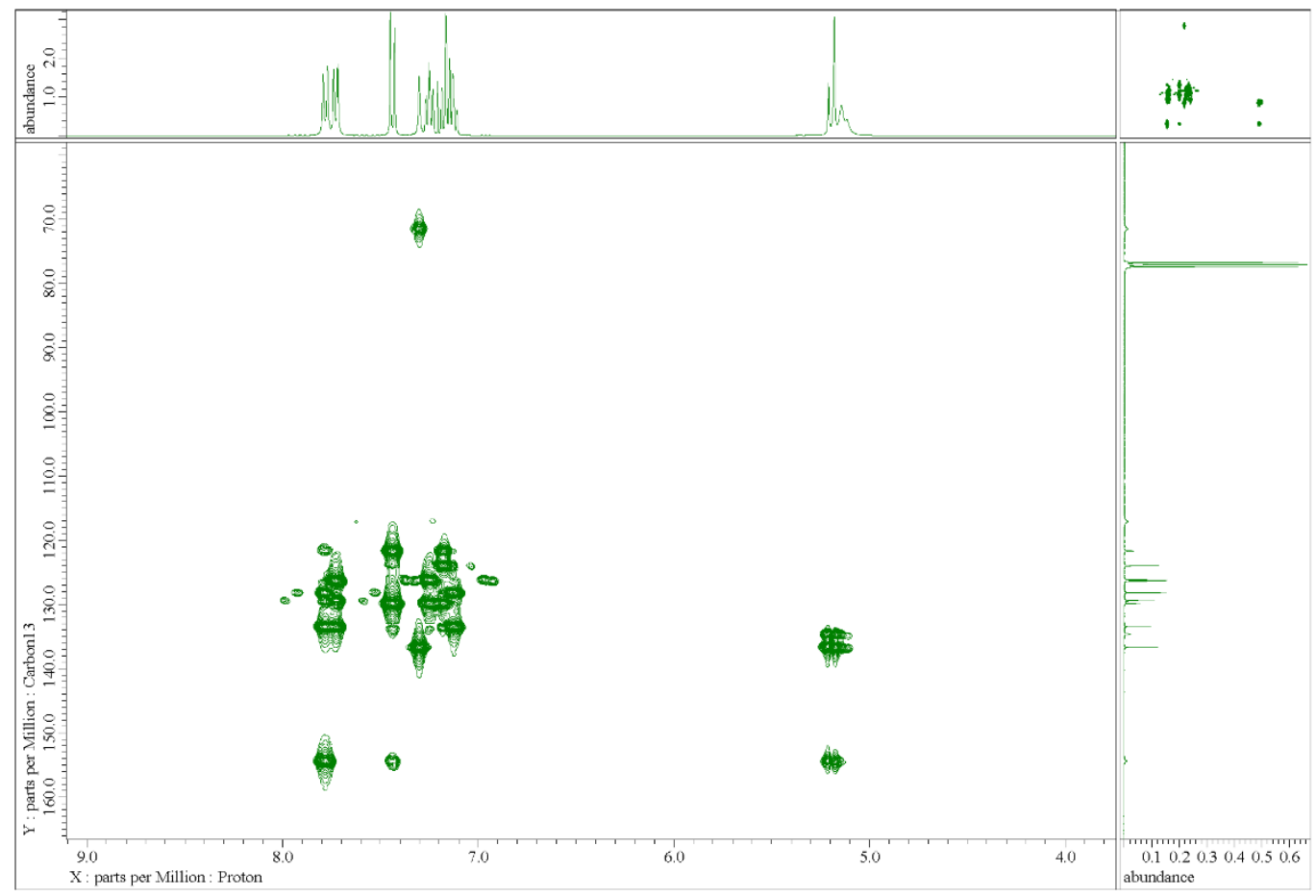

Figure S9. $H M B C$ of $B B D$. 


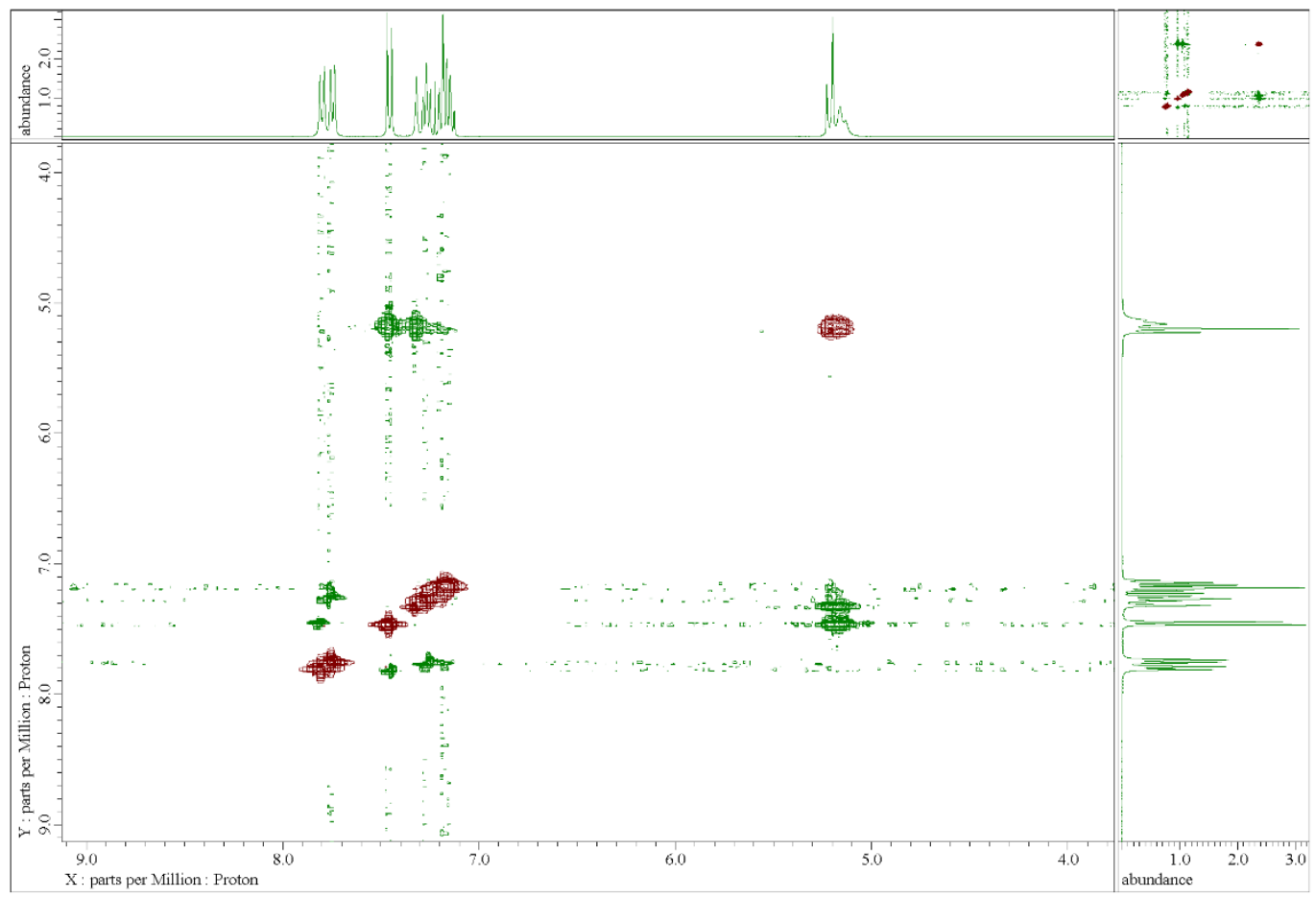

Figure S10. NOESY of BBD. 


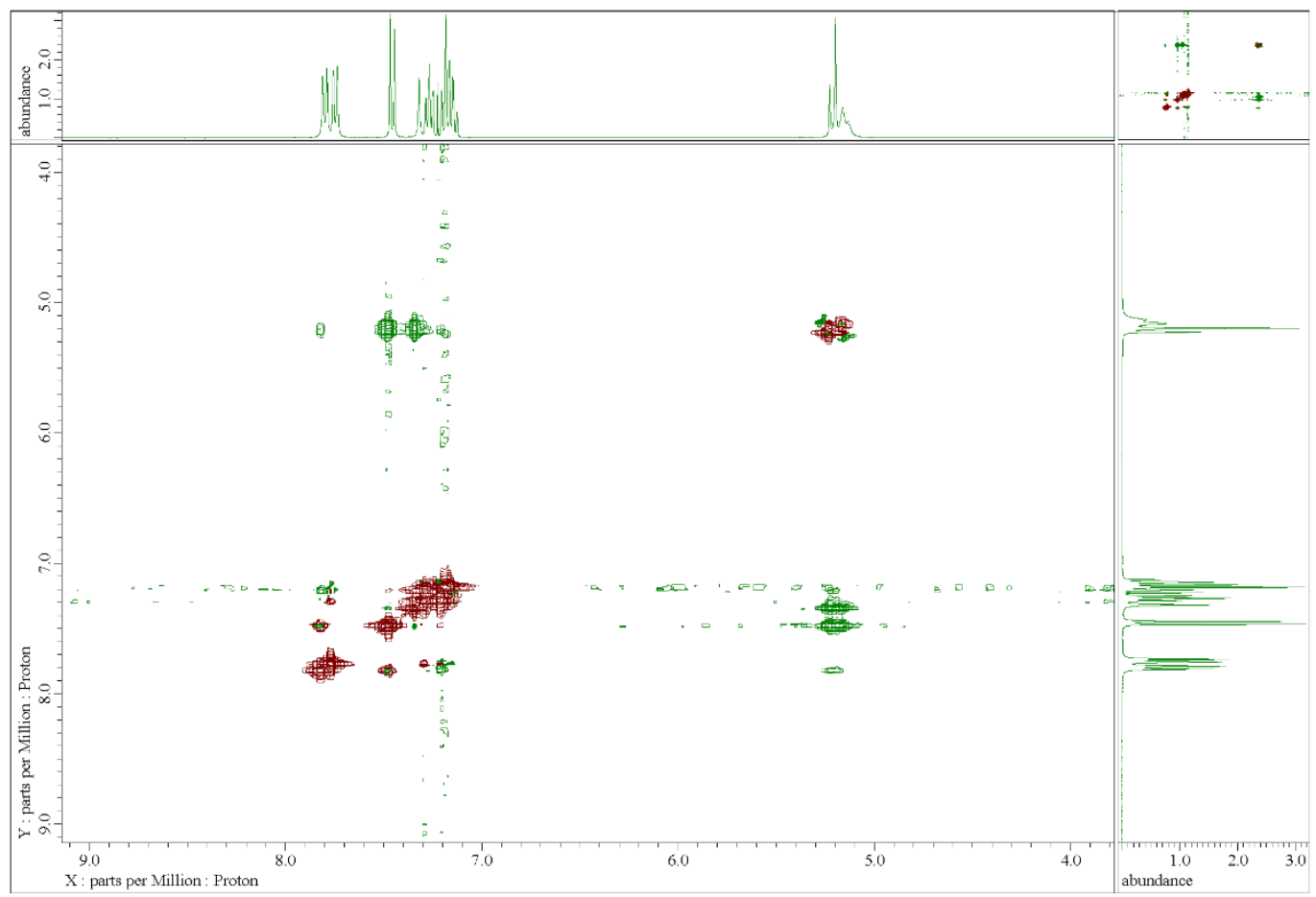

Figure S11. ROESY of BBD. 


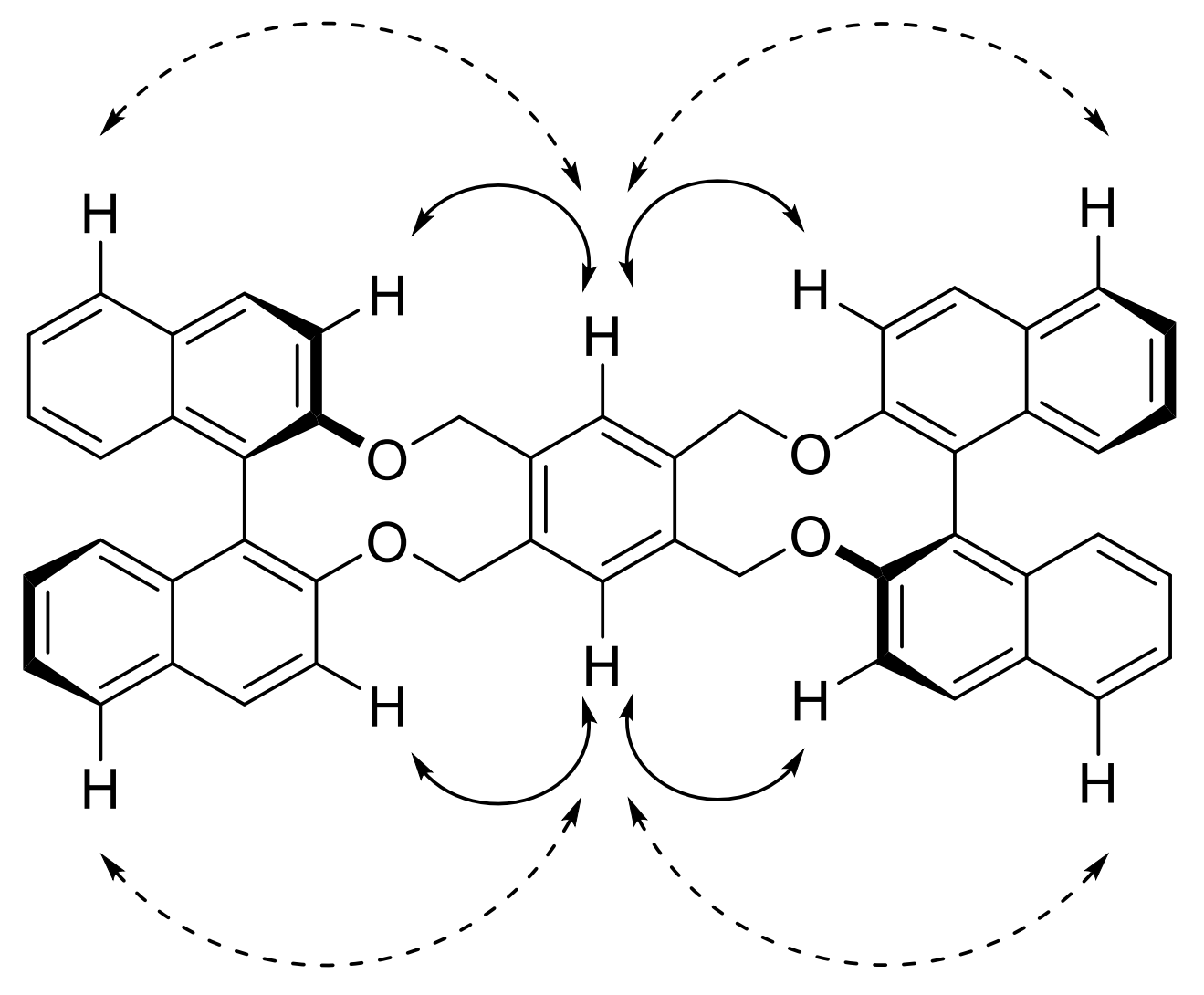

Figure S12. Selected correlation in NOESY (dashed lines) and ROESY (solid lines) spectra. 


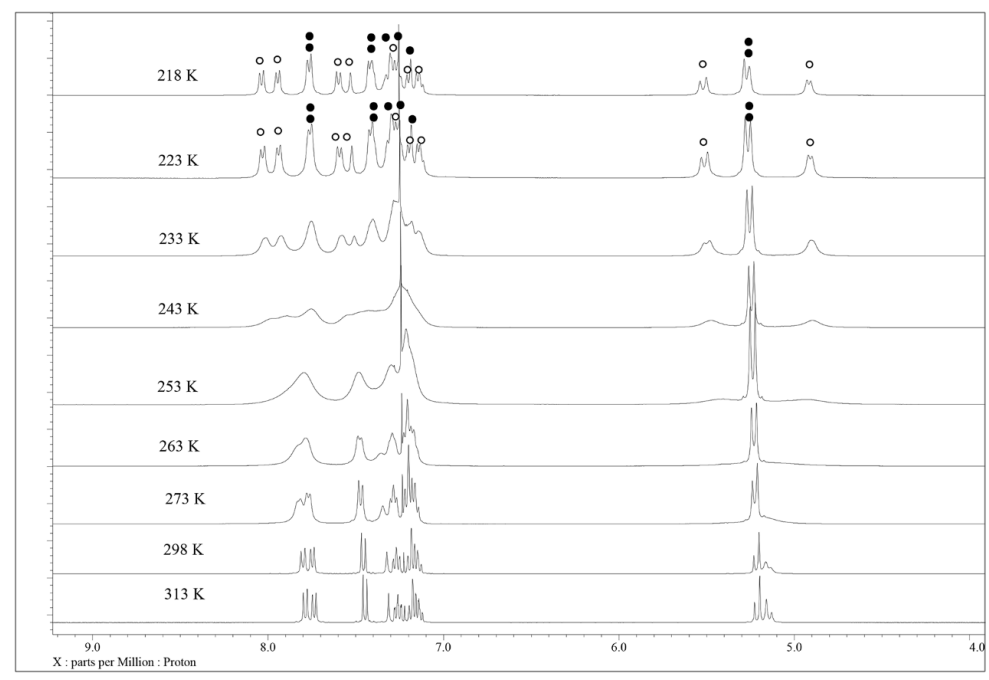

Figure S13. Temperature dependent ${ }^{1} \mathrm{H} \mathrm{NMR}\left(400 \mathrm{MHz}, \mathrm{CDCl}_{3}\right)$ spectra of $\mathrm{BBD}$. The temperature was changed from 313 to $218 \mathrm{~K}$. White and black dots at 218 and $223 \mathrm{~K}$ correspond to signals from two conformers. Proton resonances of aromatic region at the lower magnetic field were further analyzed for energetics, since they are comparably isolated. Two doublet peaks from one isomer and one overlapped doublet peaks from another isomer were observed at $218 \mathrm{~K}$, which are merged to form two doublet peaks at $313 \mathrm{~K}$.

\section{Cartesian coordinates}

See Tab. S3, Tab. S4 
a)

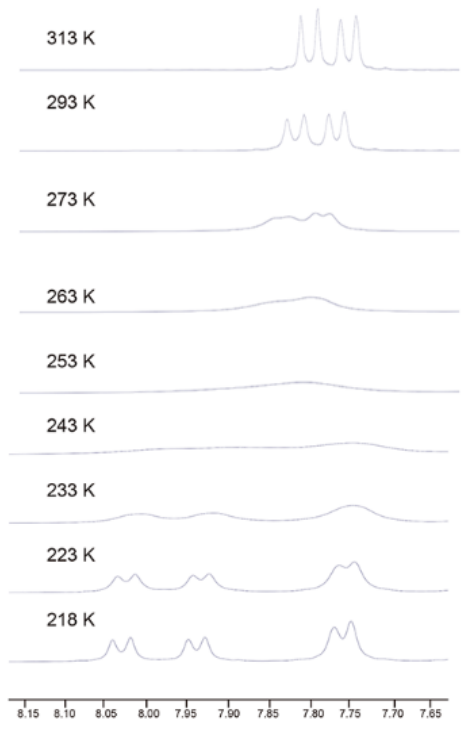

b)

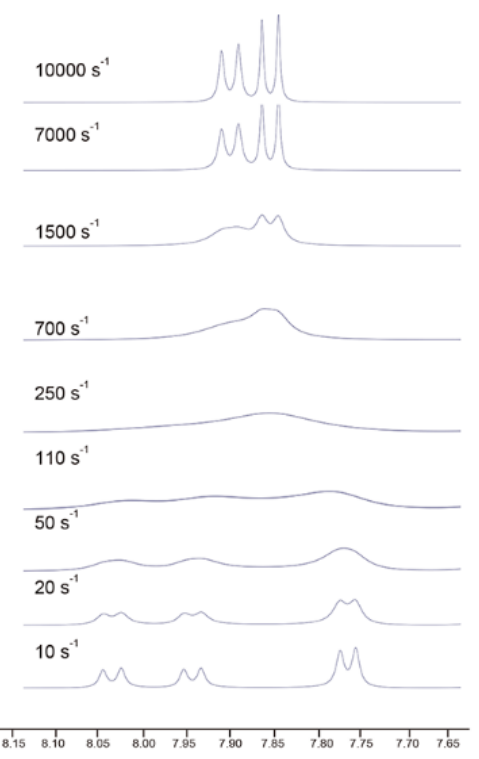

Figure S14. (a) Experimental and (b) simulated spectra of downfield region of BBD showing two sets of two aromatic proton resonances from anti and syn-isomers. One of proton sets is overlapped and apparently three peaks are shown at $218 \mathrm{~K}$. The experimental spectra were obtained in $\mathrm{CDCl}_{3}$ from 218 to $313 \mathrm{~K}$. The simulated spectra were obtained by using the designated exchange rate constants. The ratio of populations of anti and syn-isomers was set to 1. The peak-width references for the line-shape analysis were taken from the narrowest peaks (218 K for anti and syn). 


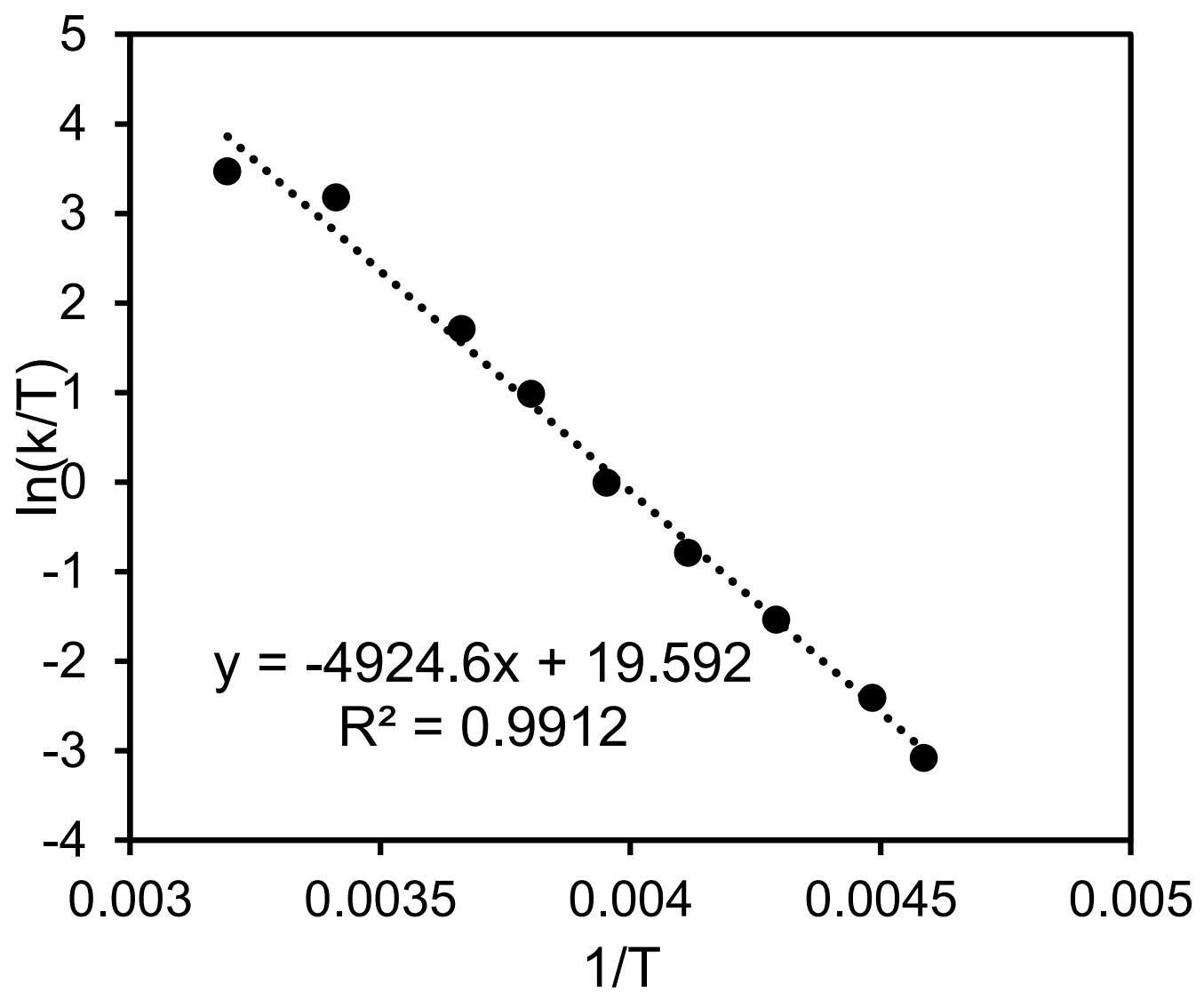

Figure S15. The Eyring plot for BBD. The energetics parameters are shown at the bottom of the plots. $\Delta H=9.8 \mathrm{kcal} / \mathrm{mol}, \Delta S=-17 \mathrm{cal} / \mathrm{mol} \mathrm{K}$.. 


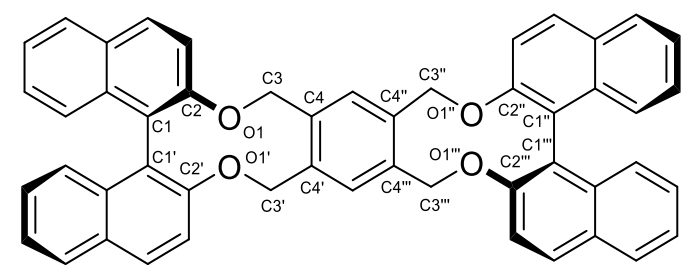

Table S1. Torsion angle of flat-, syn-, and anti-BBD optimized by DFT (B3LYP/6-31G(d,p)).

\begin{tabular}{lccr}
\hline Torsion angle & \multicolumn{1}{c}{ Flat } & \multicolumn{1}{c}{ Syn } & \multicolumn{1}{c}{ Anti } \\
\hline C2-C1-C1'-C2' $(\theta)$ & -60.5 & -63.9 & -63.9 \\
C2'-C1'-C1"-C2" $(\theta)$ & -60.5 & -63.9 & -63.9 \\
C2-O1-C3-C4 & -158.9 & -61.6 & -61.7 \\
C2'-O1'-C3'-C4' & -158.9 & -144.1 & -144.6 \\
C2"-O1"-C3"-C4" & -158.9 & -144.1 & -61.7 \\
C2'"-O1"'-C3"'-C4"' & -158.9 & -61.6 & -144.6 \\
\hline
\end{tabular}

Table S1. 


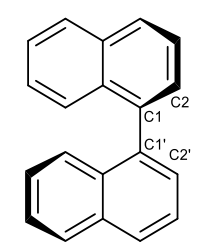

$1,1^{\prime}$-binaphthyl

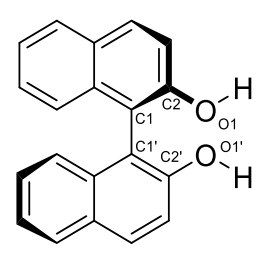

BINOL

Table S2. Torsion angle of $S_{0}$ and relaxed $S_{1}$ structures of 1,1'-binaphthyl and BINOL. Structures were optimized by DFT and TD DFT (B3LYP/6-31G(d,p)) with $C_{2}$ symmetry. Note that two conformations were obtained for 1,1'-binaphthyl but only one structure for BINOL in $\mathrm{S}_{0}$ and $\mathrm{S}_{1}$ state each.

\begin{tabular}{lrrr}
\hline & C2-C1-C1'-C2' $(\theta)$ & energy $(\mathrm{au})$ & relative energy $(\mathrm{kcal} / \mathrm{mol})$ \\
\hline 1,1'-binaphthyl $\left(\mathrm{S}_{0}\right)$ & -72.3 & -770.6099034 & 0.0 \\
1,1'-binaphthyl $\left(\mathrm{S}_{0}\right)$ & -106.8 & -770.6097537 & 0.1 \\
1,1'-binaphthyl $\left(\mathrm{S}_{1}\right)$ & -132 & -770.4794082 & 81.9 \\
1,1'-binaphthyl $\left(\mathrm{S}_{1}\right)$ & -41.4 & -770.4764297 & 83.8 \\
BINOL $\left(\mathrm{S}_{0}\right)$ & -91.1 & -921.0581789 & 0.0 \\
BINOL $\left(\mathrm{S}_{1}\right)$ & -118.6 & -920.9177153 & 88.1 \\
\hline
\end{tabular}

Table S2. 
a)

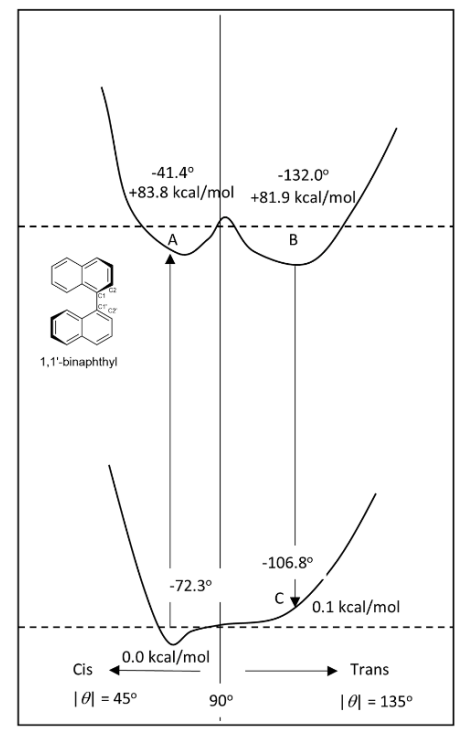

b)

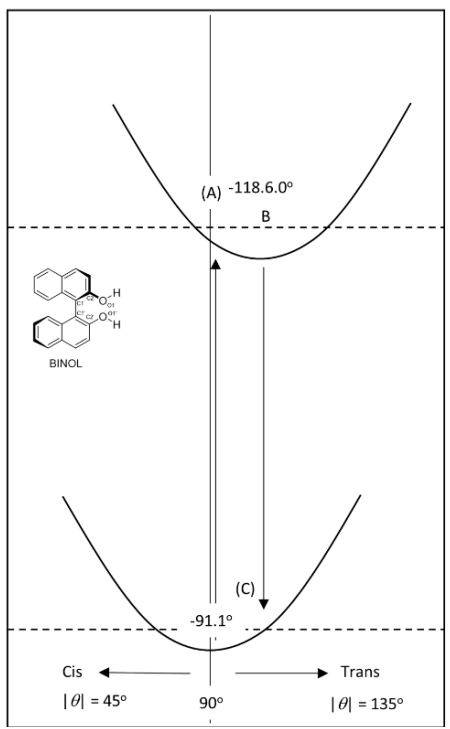

Table S16. Potential energy $\left(S_{0}\right.$ and $\left.S_{1}\right)$ of (a) 1,1'-binaphthyl and (b) BINOL, which were modified from the reported one for 1,1'-binaphthyl. ${ }^{9}$ As reported, two sets of optimized structure of 1,1'-binaphthyl were found both in $S_{0}$ and $S_{1}$ by DFT and TD-DFT (B3LYP/6-31G(d,p)) calculations. On the other hand, only one sets of optimized structure were found for BINOL by the same procedures.

\section{Theoretical calculations (MD simulations)}

\section{Structures}

The simulated systems consisted of one or two molecules on $\mathrm{Au}(111)$ 6-layer, with a vacuum layer. The size of unit cell for a single molecule on $\mathrm{Au}(111)$ was $19.9795 \times 23.0703 \times 51.7730 \AA$ and that for two molecules was $29.9693 \times 34.6055 \times 61.7730 \AA$. All simulations were performed 


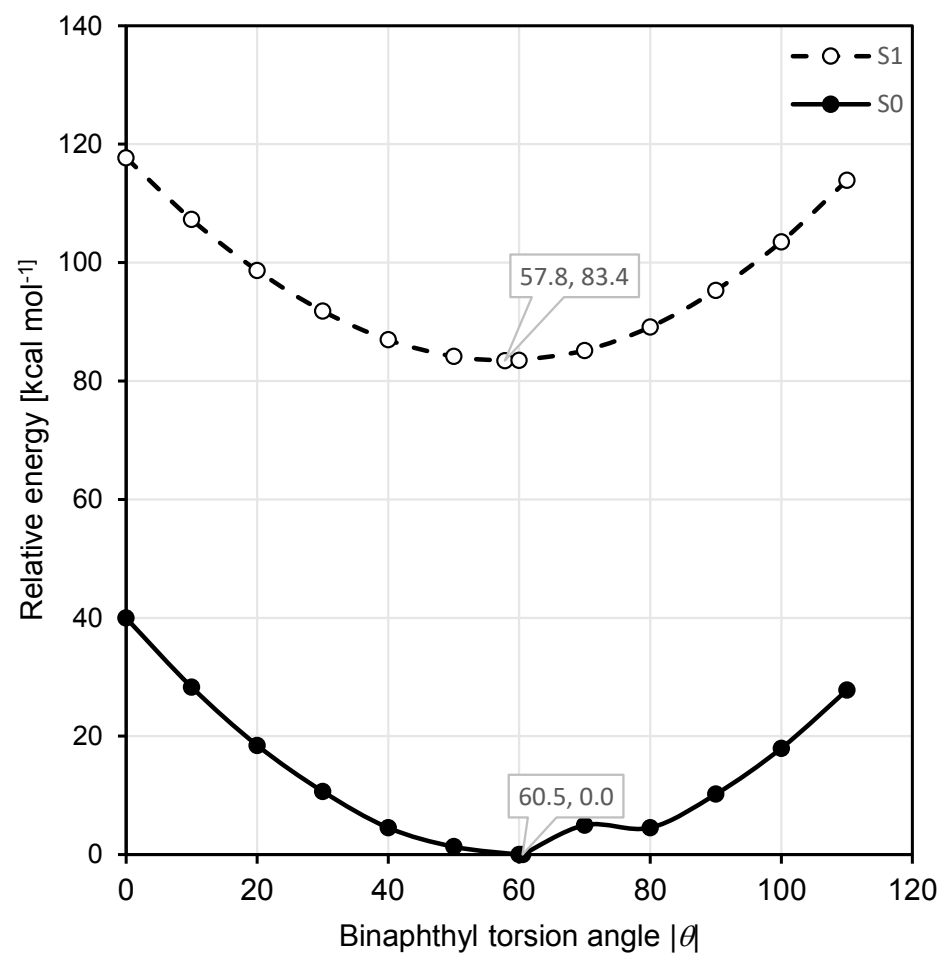

Table S17. Potential energy $\left(S_{0}\right.$ and $\left.S_{1}\right)$ of flat-BBD vs the absolute value of rbinaphthyl torsion angle $|\theta|$. The torsion angle was frozen and the structures were optimized by DFT and TD-DFT (B3LYP-6-31G(d,p)). The optimized structures without restrictions at $S_{0}$ and $S_{1}$ are also shown in the same figure and the torsion angle and the relative energy are shown respectively in the balloons. All the structures were optimized with $D_{2}$ symmetry. Only onsets of optimized structure without restriction was found in $S_{0}$ and $S_{1}$, though the local minima of $S_{0}$ at $|\theta|=80^{\circ}$ was found when the torsion angle $|\theta|$ was frozen.

in the NVT, and controlled using a NHL thermostat, with a decay constant of 1 ps.

To find several conformations of one or two molecules on Au(111) surface, anneal dynamics were used. Anneal dynamics consists of a dynamics simulation where the temperature is periodically increased from an initial temperature $(4 \mathrm{~K})$ to a mid-cycle temperature $(500 \mathrm{~K})$ and back 


\begin{tabular}{|c|c|c|c|c|c|}
\hline \multicolumn{6}{|c|}{ Table S3. Cartesian coordinate fla } \\
\hline \multirow{3}{*}{$\begin{array}{l}\text { SCF Done: } \\
-------- \\
\text { Center } \\
\text { Number }\end{array}$} & \multirow{2}{*}{$\begin{array}{l}\text { E (RB3LYP) } \\
\text { Atomic } \\
\text { Number }\end{array}$} & \multirow{2}{*}{$\begin{array}{l}-2226.7185634 \\
\begin{array}{c}\text { Atomic } \\
\text { Type }\end{array}\end{array}$} & A.U. & after 14 & ycles \\
\hline & & & \multicolumn{3}{|c|}{ Coordinates (Angstroms) } \\
\hline & & & & & \\
\hline 1 & 6 & 0 & 9.032409 & 2.403157 & -2.357358 \\
\hline 2 & 6 & 0 & 8.878316 & 2.271193 & -0.958576 \\
\hline 3 & 6 & 0 & 7.833734 & 1.546144 & -0.429263 \\
\hline 4 & 6 & 0 & 6.867277 & 0.916456 & -1.264574 \\
\hline 5 & 6 & 0 & 7.006729 & 1.094182 & -2.680638 \\
\hline 6 & 6 & 0 & 8.109094 & 1.825953 & -3.196808 \\
\hline 7 & 6 & 0 & 5.746100 & 0.172702 & $\begin{array}{l}-0.728460 \\
-0.7960\end{array}$ \\
\hline 8 & 6 & 0 & 4.750669 & -0.212848 & -1.617393 \\
\hline 9 & 6 & 0 & 4.916482 & -0.068533 & -3.020379 \\
\hline 10 & 6 & 0 & 6.024170 & 0.543910 & -3.542382 \\
\hline 11 & 6 & 0 & 5.746100 & -0.172702 & 0.728460 \\
\hline 12 & 6 & 0 & 6.867277 & -0.916456 & 1.264574 \\
\hline 13 & 6 & 0 & 7.006729 & -1.094182 & 2.680638 \\
\hline 14 & 6 & 0 & 6.024170 & -0.543910 & 3.542382 \\
\hline 15 & 6 & 0 & 4.916482 & 0.068533 & 3.020379 \\
\hline 16 & 6 & 0 & 4.750669 & 0.212848 & 1.617393 \\
\hline 17 & 6 & 0 & 7.833734 & -1.546144 & 0.429263 \\
\hline 18 & 6 & 0 & 8.878316 & -2.271193 & 0.958576 \\
\hline 19 & 6 & 0 & 9.032409 & -2.403157 & 2.357358 \\
\hline 20 & 6 & 0 & 8.109094 & -1.825953 & 3.196808 \\
\hline 21 & 8 & 0 & 3.546350 & -0.728246 & $\begin{array}{r}.170693 \\
-1.17006\end{array}$ \\
\hline 22 & 8 & 0 & 3.546350 & 0.728246 & 1.170693 \\
\hline 23 & $\begin{array}{l}8 \\
6\end{array}$ & 0 & $\begin{array}{l}3.546350 \\
2.476183\end{array}$ & $\begin{array}{l}0.128246 \\
0.173079\end{array}$ & $\begin{array}{r}1.1 \\
-1.532179\end{array}$ \\
\hline 24 & 6 & 0 & 2.476183 & -0.173079 & 1.532179 \\
\hline 25 & 6 & 0 & 1.230292 & -0.005112 & 0.701891 \\
\hline 26 & 6 & 0 & 1.230292 & 0.005112 & -0.701891 \\
\hline 27 & 6 & 0 & 0.000000 & 0.000000 & 1.365855 \\
\hline 28 & 6 & 0 & -1.230292 & 0.005112 & 0.701891 \\
\hline 29 & 6 & 0 & -1.230292 & -0.005112 & -0.701891 \\
\hline 30 & 6 & 0 & 0.000000 & 0.000000 & -1.365855 \\
\hline 31 & 6 & 0 & -9.032409 & -2.403157 & -2.357358 \\
\hline 32 & 6 & 0 & -8.878316 & -2.271193 & -0.958576 \\
\hline 33 & 6 & 0 & -7.833734 & -1.546144 & -0.429263 \\
\hline 34 & 6 & 0 & -6.867277 & -0.916456 & -1.264574 \\
\hline 35 & 6 & 0 & -7.006729 & -1.094182 & -2.680638 \\
\hline 36 & 6 & 0 & -8.109094 & -1.825953 & -3.196808 \\
\hline 37 & 6 & 0 & -5.746100 & -0.172702 & -0.728460 \\
\hline 38 & $\begin{array}{l}6 \\
6\end{array}$ & 0 & -4.750669 & 0.212848 & $\begin{array}{r}-0.1617390 \\
-1.61739\end{array}$ \\
\hline $\begin{array}{l}38 \\
39\end{array}$ & $\begin{array}{l}6 \\
6\end{array}$ & 0 & $\begin{array}{r}-4.150669 \\
-4.916482\end{array}$ & 0.068533 & $\begin{array}{l}-1.61939 \\
-3.020379\end{array}$ \\
\hline 40 & 6 & 0 & -6.024170 & -0.543910 & -3.542382 \\
\hline 41 & 6 & 0 & -5.746100 & 0.172702 & 0.728460 \\
\hline 42 & 6 & 0 & -6.867277 & 0.916456 & 1.264574 \\
\hline 43 & 6 & 0 & -7.006729 & 1.094182 & 2.680638 \\
\hline 44 & 6 & 0 & -6.024170 & 0.543910 & 3.542382 \\
\hline 45 & 6 & 0 & -4.916482 & -0.068533 & 3.020379 \\
\hline 46 & 6 & 0 & -4.750669 & -0.212848 & 1.617393 \\
\hline 47 & 6 & 0 & -7.833734 & 1.546144 & 0.429263 \\
\hline 48 & 6 & 0 & -8.878316 & 2.271193 & 0.958576 \\
\hline 49 & 6 & 0 & -9.032409 & 2.403157 & 2.357358 \\
\hline 50 & 6 & 0 & -8.109094 & 1.825953 & 3.196808 \\
\hline 51 & 8 & 0 & -3.546350 & 0.728246 & -1.170693 \\
\hline 52 & 8 & 0 & -3.546350 & -0.728246 & $\begin{array}{r}1.170693 \\
\end{array}$ \\
\hline 53 & 6 & 0 & -2.476183 & -0.173079 & $\begin{array}{r}-1.532179 \\
\end{array}$ \\
\hline 54 & $\begin{array}{l}0 \\
6\end{array}$ & 0 & -2.476183 & $\begin{array}{r}-173079 \\
0.173079\end{array}$ & $\begin{array}{r}-1.532179 \\
1.532179\end{array}$ \\
\hline $\begin{array}{l}54 \\
55\end{array}$ & $\begin{array}{l}6 \\
1 \\
1\end{array}$ & 0 & $\begin{array}{r}-2.476183 \\
9.866946\end{array}$ & 2.967985 & $\begin{array}{r}1.532189 \\
-2.761895\end{array}$ \\
\hline 56 & 1 & 0 & 9.589560 & 2.749545 & -0.291520 \\
\hline 57 & 1 & 0 & 7.732782 & 1.468743 & 0.645872 \\
\hline 58 & 1 & 0 & 8.198273 & 1.932890 & -4.274840 \\
\hline 59 & 1 & 0 & 4.147648 & -0.470639 & -3.671716 \\
\hline 60 & 1 & 0 & 6.148802 & 0.636520 & -4.617844 \\
\hline 61 & 1 & 0 & 6.148802 & -0.636520 & 4.617844 \\
\hline 62 & 1 & 0 & 4.147648 & 0.470639 & 3.671716 \\
\hline 63 & 1 & 0 & 7.732782 & -1.468743 & -0.645872 \\
\hline 64 & 1 & 0 & 9.589560 & -2.749545 & 0.291520 \\
\hline 65 & 1 & 0 & 9.866946 & -2.967985 & 2.761895 \\
\hline 66 & 1 & 0 & 8.198273 & -1.932890 & 4.274840 \\
\hline 67 & 1 & 0 & 2.839133 & 1.200244 & -1.410151 \\
\hline 68 & 1 & 0 & 2.219323 & 0.033484 & -2.589136 \\
\hline
\end{tabular}

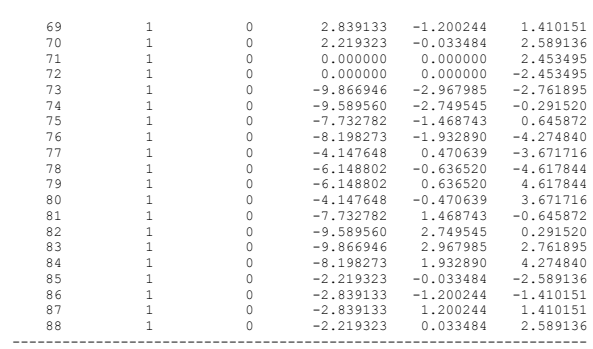

Table S3. 
Table S4. Cartesian coordinate syn-conformer

\begin{tabular}{|c|c|c|c|c|c|}
\hline \multirow{2}{*}{$\begin{array}{l}\text { Center } \\
\text { Number }\end{array}$} & \multirow{2}{*}{$\begin{array}{l}\text { Atomic } \\
\text { Number }\end{array}$} & \multirow{2}{*}{$\begin{array}{c}\text { Atomic } \\
\text { Type }\end{array}$} & \multicolumn{3}{|c|}{ Coordinates (Angstroms) } \\
\hline & & & $\mathrm{x}$ & $\mathrm{Y}$ & Z \\
\hline 1 & 6 & 0 & -0.733469 & 3.186147 & 4.604041 \\
\hline 2 & 6 & 0 & 0.165234 & 4.130915 & 4.053036 \\
\hline 3 & 6 & 0 & 0.190067 & 4.372704 & 2.698304 \\
\hline 4 & 6 & 0 & -0.685194 & 3.683156 & 1.810751 \\
\hline 5 & 6 & 0 & -1.576897 & 2.708957 & 2.372026 \\
\hline 6 & 6 & 0 & -1.581558 & 2.489774 & 3.775856 \\
\hline 7 & 6 & 0 & -0.676896 & 3.903212 & 0.395108 \\
\hline 8 & 6 & 0 & -1.508763 & 3.131142 & -0.408429 \\
\hline 9 & 6 & 0 & -2.387533 & 2.170286 & 0.146395 \\
\hline 10 & 6 & 0 & -2.421343 & 1.972640 & 1.505373 \\
\hline 11 & 6 & 0 & 0.233732 & 4.900790 & -0.243211 \\
\hline 12 & 6 & 0 & 0.080344 & 6.313333 & -0.042731 \\
\hline 13 & 6 & 0 & 0.985718 & 7.224293 & -0.684228 \\
\hline 14 & 6 & 0 & 2.011291 & 6.707653 & -1.516991 \\
\hline 15 & 6 & 0 & 2.133458 & 5.355253 & -1.721371 \\
\hline 16 & 6 & 0 & 1.240715 & 4.456071 & -1.089081 \\
\hline 17 & 6 & 0 & -0.969759 & 6.862759 & 0.746228 \\
\hline 18 & 6 & 0 & -1.100635 & 8.223272 & 0.910474 \\
\hline 19 & 6 & 0 & -0.190067 & 9.116203 & 0.297153 \\
\hline 20 & 6 & 0 & 0.827527 & 8.622124 & -0.484786 \\
\hline 21 & 8 & 0 & -1.465688 & 3.388694 & -1.759895 \\
\hline 22 & 8 & 0 & 1.382037 & 3.100685 & -1.280531 \\
\hline 23 & 6 & 0 & -1.758151 & 2.345100 & -2.688229 \\
\hline 24 & 6 & 0 & 1.267869 & 2.636378 & -2.638655 \\
\hline 25 & 6 & 0 & 0.581349 & 1.289764 & -2.641528 \\
\hline 26 & 6 & 0 & -0.819696 & 1.153553 & -2.630881 \\
\hline 27 & 6 & 0 & 1.364884 & 0.133016 & -2.630549 \\
\hline 28 & 6 & 0 & 0.819696 & -1.153553 & -2.630881 \\
\hline 29 & 6 & 0 & -0.581349 & -1.289764 & -2.641528 \\
\hline 30 & 6 & 0 & -1.364884 & -0.133016 & -2.630549 \\
\hline 31 & 6 & 0 & 0.190067 & -9.116203 & 0.297153 \\
\hline 32 & 6 & 0 & 1.100635 & -8.223272 & 0.910474 \\
\hline 33 & 6 & 0 & 0.969759 & -6.862759 & 0.746228 \\
\hline 34 & 6 & 0 & -0.080344 & -6.313333 & -0.042731 \\
\hline 35 & 6 & 0 & -0.985718 & -7.224293 & -0.684228 \\
\hline 36 & 6 & 0 & -0.827527 & -8.622124 & -0.484786 \\
\hline 37 & 6 & 0 & -0.233732 & -4.900790 & -0.243211 \\
\hline 38 & 6 & 0 & -1.240715 & -4.456071 & -1.089081 \\
\hline 39 & 6 & 0 & -2.133458 & -5.355253 & -1.721371 \\
\hline 40 & 6 & 0 & -2.011291 & -6.707653 & -1.516991 \\
\hline 41 & 6 & 0 & 0.676896 & -3.903212 & 0.395108 \\
\hline 42 & 6 & 0 & 0.685194 & -3.683156 & 1.810751 \\
\hline 43 & 6 & 0 & 1.576897 & -2.708957 & 2.372026 \\
\hline 44 & 6 & 0 & 2.421343 & -1.972640 & 1.505373 \\
\hline 45 & 6 & 0 & 2.387533 & -2.170286 & 0.146395 \\
\hline 46 & 6 & 0 & 1.508763 & -3.131142 & -0.408429 \\
\hline 47 & 6 & 0 & -0.190067 & -4.372704 & 2.698304 \\
\hline 48 & 6 & 0 & -0.165234 & -4.130915 & 4.053036 \\
\hline
\end{tabular}

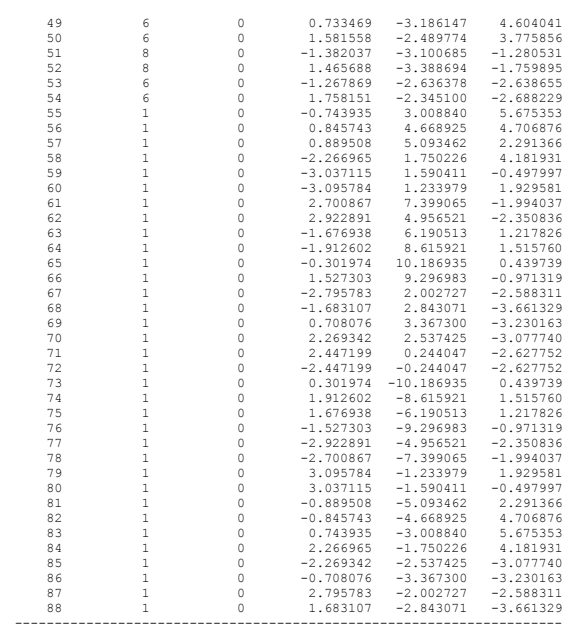

Table S4. 


\begin{tabular}{|c|c|c|c|c|c|}
\hline $\begin{array}{l}\text { Center } \\
\text { Number }\end{array}$ & $\begin{array}{l}\text { Atomic } \\
\text { Number }\end{array}$ & $\begin{array}{c}\text { Atomic } \\
\text { Type }\end{array}$ & \multicolumn{3}{|c|}{ Coordinates (Angstroms) } \\
\hline 1 & 6 & 0 & -1.387323 & 9.454777 & -0.554064 \\
\hline${ }_{3}^{2}$ & $\begin{array}{l}6 \\
6\end{array}$ & $\begin{array}{l}0 \\
0\end{array}$ & $\begin{array}{l}-0.498019 \\
-0.041161\end{array}$ & $\begin{array}{r}9.004789 \\
7.706111\end{array}$ & $\begin{array}{l}0.450489 \\
0.454733\end{array}$ \\
\hline $\begin{array}{l}3 \\
4\end{array}$ & $\begin{array}{l}6 \\
6\end{array}$ & $\begin{array}{l}0 \\
0\end{array}$ & $\begin{array}{l}-0.0041161 \\
-0.447367\end{array}$ & $\begin{array}{l}7.706116 \\
6.778844\end{array}$ & $\begin{array}{r}0.4447333 \\
-0.546396\end{array}$ \\
\hline 5 & 6 & 0 & -1.370609 & 7.232801 & -1.547665 \\
\hline 6 & 6 & 0 & -1.813864 & 8.582540 & -1.527893 \\
\hline 7 & 6 & 0 & 0.000000 & 5.415425 & -0.560755 \\
\hline & $\begin{array}{l}6 \\
6\end{array}$ & $\begin{array}{l}0 \\
0\end{array}$ & $\begin{array}{l}-0.5000033 \\
-1.416935\end{array}$ & $\begin{array}{l}4.559991 \\
5.000126\end{array}$ & $\begin{array}{l}-1.529517 \\
-2.551739\end{array}$ \\
\hline 10 & 6 & $\begin{array}{l}0 \\
0\end{array}$ & $\begin{array}{l}-1.446935 \\
-1.832884\end{array}$ & $\begin{array}{l}5.009126 \\
6.317673\end{array}$ & $\begin{array}{l}-2.551391 \\
-2.528028\end{array}$ \\
\hline 11 & 6 & 0 & 0.955771 & $\begin{array}{l}4.881767 \\
5.315452\end{array}$ & 0.456002 \\
\hline 12 & $\begin{array}{l}6 \\
6\end{array}$ & ${ }_{0}^{0}$ & $\begin{array}{l}2.3200044 \\
3.195758\end{array}$ & $\begin{array}{l}5.3155452 \\
4.776160\end{array}$ & $\begin{array}{l}0.513566 \\
1.55457\end{array}$ \\
\hline $\begin{array}{l}13 \\
14\end{array}$ & 6 & 0 & $\begin{array}{r}1.195158 \\
2.689930\end{array}$ & 3.808835 & $\begin{array}{l}1.5514576 \\
2.41721\end{array}$ \\
\hline 15 & 6 & 0 & $\begin{array}{l}1.388904 \\
\end{array}$ & 3.375114 & 2.335350 \\
\hline 16 & 6 & 0 & 0.523932 & 3.906102 & 1.348235 \\
\hline $\begin{array}{l}17 \\
18\end{array}$ & 6 & 0 & $\begin{array}{l}2.866294 \\
4\end{array}$ & $\begin{array}{l}6.248417 \\
6 \\
6.639337\end{array}$ & $\begin{array}{l}-0.414042 \\
-0.3868\end{array}$ \\
\hline & & 0 & $\begin{array}{l}4.183773 \\
5.03641\end{array}$ & $\begin{array}{l}6.6393144 \\
6.123192\end{array}$ & $\begin{array}{r}-0.338680 \\
0.666625\end{array}$ \\
\hline 20 & 6 & 0 & 4.548268 & $\begin{array}{l}0.119 \\
5.208195\end{array}$ & $\begin{array}{l}0.666625 \\
1.569115\end{array}$ \\
\hline 21 & 8 & 0 & -0.077011 & 3.253545 & -1.534213 \\
\hline 22 & 8 & 0 & -0.795652 & 3.529859 & 1.255235 \\
\hline $\begin{array}{l}23 \\
24\end{array}$ & 6 & 0 & -1.100303 & 2.246381 & -1.409707 \\
\hline $\begin{array}{l}24 \\
25\end{array}$ & $\begin{array}{l}6 \\
6\end{array}$ & 0 & $\begin{array}{l}-1.18161660 \\
-0.549146\end{array}$ & $\begin{array}{l}2.207409 \\
1.097482\end{array}$ & $\begin{array}{l}1.6298839 \\
0.810433\end{array}$ \\
\hline 26 & 6 & 0 & -0.551683 & 1.096522 & -0.597265 \\
\hline $\begin{array}{l}27 \\
28\end{array}$ & $\begin{array}{l}6 \\
6\end{array}$ & $\begin{array}{l}0 \\
0\end{array}$ & $\begin{array}{l}0.0000000 \\
0.549146\end{array}$ & $\begin{array}{r}0.0000000 \\
-1.097482\end{array}$ & $\begin{array}{l}1.4788000 \\
0.810433\end{array}$ \\
\hline
\end{tabular}

\begin{tabular}{|c|c|c|c|c|c|}
\hline 29 & 6 & 0 & 0.551683 & -1.096522 & -0.597265 \\
\hline 30 & 6 & 0 & 0.000000 & 0.000000 & -1.264098 \\
\hline 31 & 6 & 0 & 1.387323 & -9.454777 & -0.554064 \\
\hline 32 & 6 & 0 & 0.498019 & -9.004789 & 0.450489 \\
\hline 33 & 6 & 0 & 0.041161 & -7.706116 & 0.454733 \\
\hline 34 & 6 & 0 & 0.447367 & -6.778844 & -0.546396 \\
\hline 35 & 6 & 0 & 1.370609 & -7.232801 & -1.547665 \\
\hline 36 & 6 & 0 & 1.813864 & -8.582540 & -1.527893 \\
\hline 37 & 6 & 0 & 0.000000 & -5.415425 & -0.560755 \\
\hline 38 & 6 & 0 & 0.507033 & -4.559791 & -1.529517 \\
\hline 39 & 6 & 0 & 1.416935 & -5.009126 & -2.517391 \\
\hline 40 & 6 & 0 & 1.832884 & -6.317673 & -2.528028 \\
\hline 41 & 6 & 0 & -0.955771 & -4.881767 & 0.456002 \\
\hline 42 & 6 & 0 & -2.320044 & -5.315452 & 0.513566 \\
\hline 43 & 6 & 0 & -3.195758 & -4.776160 & 1.514576 \\
\hline 44 & 6 & 0 & -2.689930 & -3.808835 & 2.417211 \\
\hline 45 & 6 & 0 & -1.388904 & -3.375114 & 2.335350 \\
\hline 46 & 6 & 0 & -0.523932 & -3.906102 & 1.348235 \\
\hline 47 & 6 & 0 & -2.866294 & -6.248417 & -0.414042 \\
\hline 48 & 6 & 0 & -4.183773 & -6.639334 & -0.338680 \\
\hline 49 & 6 & 0 & -5.036416 & -6.123192 & 0.666625 \\
\hline 50 & 6 & 0 & -4.548268 & -5.208195 & 1.569115 \\
\hline 51 & 8 & 0 & 0.077011 & -3.253545 & -1.534213 \\
\hline 52 & 8 & 0 & 0.795652 & -3.529859 & 1.255235 \\
\hline 53 & 6 & 0 & 1.100303 & -2.246381 & -1.409707 \\
\hline 54 & 6 & 0 & 1.181660 & -2.207409 & 1.629839 \\
\hline 55 & 1 & 0 & -1.735340 & 10.483440 & -0.548297 \\
\hline 56 & 1 & 0 & -0.174187 & 9.691114 & 1.227614 \\
\hline 57 & 1 & 0 & 0.635523 & 7.373486 & 1.233050 \\
\hline 58 & 1 & 0 & -2.506360 & 8.911410 & -2.298675 \\
\hline 59 & 1 & 0 & -1.761821 & 4.309155 & -3.271897 \\
\hline 60 & 1 & 0 & -2.524368 & 6.667370 & -3.289984 \\
\hline 61 & 1 & 0 & 3.350272 & 3.404167 & 3.179630 \\
\hline 62 & 1 & 0 & 1.023987 & 2.632113 & 3.034532 \\
\hline 63 & 1 & 0 & 2.228678 & 6.647558 & -1.194089 \\
\hline 64 & 1 & 0 & 4.574959 & 7.349835 & -1.061206 \\
\hline 65 & 1 & 0 & 6.072371 & 6.444922 & 0.716407 \\
\hline 66 & 1 & 0 & 5.193777 & 4.793441 & 2.339298 \\
\hline 67 & 1 & 0 & -1.985741 & 2.681667 & -0.936416 \\
\hline 68 & 1 & 0 & -1.386629 & 1.887462 & -2.406481 \\
\hline 69 & 1 & 0 & -1.000996 & 2.027948 & 2.697132 \\
\hline 70 & 1 & 0 & -2.268208 & 2.210939 & 1.490691 \\
\hline 71 & 1 & 0 & 0.000000 & 0.000000 & 2.567186 \\
\hline 72 & 1 & 0 & 0.000000 & 0.000000 & -2.351632 \\
\hline 73 & 1 & 0 & 1.735340 & -10.483440 & -0.548297 \\
\hline 74 & 1 & 0 & 0.174187 & -9.691114 & 1.227614 \\
\hline 75 & 1 & 0 & -0.635523 & -7.373486 & 1.233050 \\
\hline 76 & 1 & 0 & 2.506360 & -8.911410 & -2.298675 \\
\hline 77 & 1 & 0 & 1.761821 & -4.309155 & -3.271897 \\
\hline 78 & 1 & 0 & 2.524368 & -6.667370 & -3.289984 \\
\hline 79 & 1 & 0 & -3.350272 & -3.404167 & 3.179630 \\
\hline 80 & 1 & 0 & -1.023987 & -2.632113 & 3.034532 \\
\hline 81 & 1 & 0 & -2.228678 & -6.647558 & -1.194089 \\
\hline 82 & 1 & 0 & -4.574959 & -7.349835 & -1.061206 \\
\hline 83 & 1 & 0 & -6.072371 & -6.444922 & 0.716407 \\
\hline 84 & 1 & 0 & -5.193777 & -4.793441 & 2.339298 \\
\hline 85 & 1 & 0 & 1.386629 & -1.887462 & -2.406481 \\
\hline 86 & 1 & 0 & 1.985741 & -2.681667 & -0.936416 \\
\hline 87 & 1 & 0 & 1.000996 & -2.027948 & 2.697132 \\
\hline 88 & 1 & 0 & 2.268208 & -2.210939 & 1.490691 \\
\hline
\end{tabular}

Table S5. 
again. All MD calculations were performed with Forcite in Materials Studio, and force field COMPASS was used. All the structures obtained were optimized with the same force field.

For calculations of a monomer on $\mathrm{Au}(111)$, the DFT optimized anti-structure was used as an initial structure, and syn-conformer on $\mathrm{Au}(111)$ surface was obtained as a stable conformer. The anti-conformer on $\mathrm{Au}(111)$ surface was also obtained with higher energy $(+9.0 \mathrm{kcal} / \mathrm{mol})$. When a mid-cycle temperature was raised to $1500 \mathrm{~K}$, flat-conformer was found with lower energy, $-22.5 \mathrm{kcal} / \mathrm{mol}$ compared with the syn-conformer. For calculations of a dimer on $\mathrm{Au}(111)$, the DFT optimized two syn-conformers were used as initial structures, and syn-syn-dimer on $\mathrm{Au}(111)$ surface was obtained as a stable conformer.

See Fig. S18 and Fig. S19.

\section{Interaction energies}

Each total energies of syn-, anti-, and flat-conformers on $\mathrm{Au}(111)$ surface $\left(E_{\text {total_syn }}, E_{\text {total_anti, }}\right.$, and $\left.E_{\text {total_flat }}\right)$ were $-19016.6,-19007.6$, and $-19039.0 \mathrm{kcal} / \mathrm{mol}$, respectively. The following energies were obtained as a single point energy calculation with the same force field.

The energy of $\mathrm{Au}(111)\left(E_{\text {surface_monomer }}\right)$ was $-19536.0 \mathrm{kcal} / \mathrm{mol}$, those of syn-, anti-, and flat- conformers $\left(E_{\text {rmsyn }}, E_{\text {rmanti }}\right.$, and $E_{\text {rmflat }}$, respectively) were $714.3,722.7$, and $721.2 \mathrm{kcal} / \mathrm{mol}$, respectively.

The interaction energy between $\mathrm{Au}(111)$ and syn--conformer ( $\left.E_{\text {surfaceinteraction_syn }}\right)$ was calculated to be $-194.92 \mathrm{kcal} / \mathrm{mol}$ based on the following equation:

$$
E_{\text {surfaceinteraction_syn }}=E_{\text {total_syn }}-\left(E_{\text {surface_monomer }}+E_{\text {syn }}\right) \text {. }
$$

The interaction energy between $\mathrm{Au}(111)$ and anti- and flat-conformer ( $E_{\text {surfaceinteraction_anti }}$, $\left.E_{\text {surfaceinteraction_flat }}\right)$ was calculated with the same method to be -194.3 and $-244.2 \mathrm{kcal} / \mathrm{mol}$, respectively.

The surface interaction energy for syn- or anti-conformers were at the same level $\left(E_{\text {surfaceinteraction_syn }}=\right.$ $\left.-194.9 \mathrm{kcal} / \mathrm{mol}, E_{\text {surfaceinteraction_anti }}=-194.3 \mathrm{kcal} / \mathrm{mol}\right)$. The difference in stability of synand anti-conformers on $\mathrm{Au}(111)$ originates from the difference in molecular deformation en$\operatorname{ergy}\left(E_{\text {anti }}-E_{\text {syn }}=+8.4 \mathrm{kcal} / \mathrm{mol}\right.$ higher than syn-conformer $)$ rather than interaction energy ( $E_{\text {surfaceinteraction_anti }}-E_{\text {surfaceinteraction_syn }}=+0.6 \mathrm{kcal} / \mathrm{mol}$ more unfavorable for the anti-conformer). In another words, to obtain similar interaction with $\mathrm{Au}(111)$ surface, anti-conformer needed to be 
deformed. On the other hand, the stability of flat-conformer mainly originate from the large surface interaction energy $\left(E_{\text {surfaceinteraction_flat }}-E_{\text {surfaceinteraction_syn }}=-29.3 \mathrm{kcal} / \mathrm{mol}\right.$ more favorable than syn-conformer) rather than molecular deformation energy $\left(E_{\mathrm{flat}}-E_{\mathrm{syn}}=+6.9 \mathrm{kcal} / \mathrm{mol}\right.$ higher than syn-conformer).

Total energy of syn-syn dimer on $\mathrm{Au}(111)$ surface $\left(E_{\text {total }}\right)$ was $-42912.6 \mathrm{kcal} / \mathrm{mol}$. The following energies were obtained as a single point energy calculation with the same force field. The energy of $\mathrm{Au}(111)\left(E_{\text {surface }}\right)$ was $-43945.7 \mathrm{kcal} / \mathrm{mol}$, that of syn-syn $\operatorname{dimer}\left(E_{\text {syn-syn-dimer }}\right)$ is 1438.2 $\mathrm{kcal} / \mathrm{mol}$, those of each syn-conformers $\left(E_{\text {syn-conformer-1 }}\right.$ and $\left.E_{\text {syn-conformer-2 }}\right)$ are $724.3 \mathrm{kcal} / \mathrm{mol}$ and 724.3 , respectively. The interaction energy between $\mathrm{Au}(111)$ and syn-syn dimers $\left(E_{\text {surfaceinteraction }}\right)$ was calculated to be $-405.1 \mathrm{kcal} / \mathrm{mol}$ and that between syn-syn dimers ( $\left.E_{\text {molecularinteraction }}\right)$ was to be $-10.4 \mathrm{kcal} / \mathrm{mol}$ based on the following equations:

$$
\begin{gathered}
E_{\text {surfaceinteraction }}=E_{\text {total }}-\left(E_{\text {surface }}+E_{\text {syn-syn-dimer }}\right) \\
E_{\text {molecularinteraction }}=E_{\text {syn-syn-dimer }}-\left(E_{\text {syn-conformer-1 }}+E_{\text {syn-conformer-2 }}\right)
\end{gathered}
$$

The formation of syn-syn dimer is more favorable than being two syn-monomers, by the energy of $E=\left(E_{\text {total }}-E_{\text {surface }}\right) / 2-\left(E_{\text {total }_{\mathrm{y} y n}}-E_{\text {surface }_{\mathrm{m}} \text { onomer }}\right)=-2.8 \mathrm{kcal} / \mathrm{mol}$. The formation of syn-syn dimer (in vacuum) is estimated to be $E_{\text {syn-syn-dimer }} / 2-E s y n=+4.8 \mathrm{kcal} / \mathrm{mol}$ more unfavorable compared with being monomers. On the other hand, surface interaction energy became $E_{\text {surfaceinteraction }} / 2-E_{\text {surfaceinteraction }_{\mathrm{s}} \mathrm{yn}}=-7.6 \mathrm{kcal} / \mathrm{mol}$ more favorable for formation of dimers. As a result, each syn-conformers are $E=-7.6+4.8=-2.8 \mathrm{kcal} / \mathrm{mol}$ more favorable as a dimer compared with being monomers.

\section{Conformation transformations by forcible molecule manipulation}

Here we provide more details and examples of lateral single molecule manipulation protocol in a mechanical mode to succeed to prepare one by one single planar BBD molecules are presented and used in the manuscript.

See Fig. S20.

\section{Inelastic electronic tunneling effect}

After some of the inelastic electronic tunneling excitation of a BBD molecule by locating the STM tip apex on the highest electronic probability density site of a BBD molecule, we have 
a)

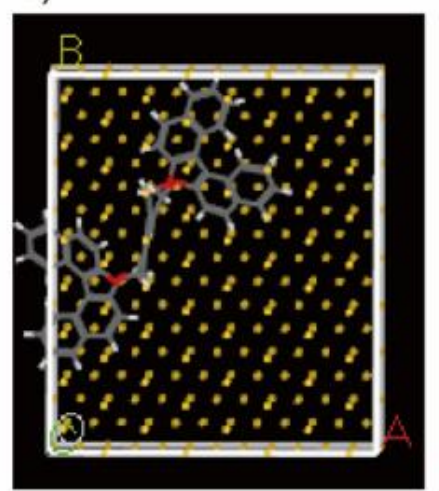

d)

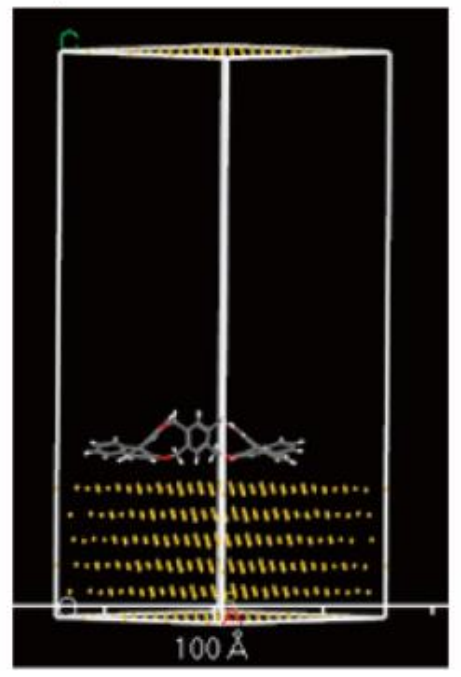

b)

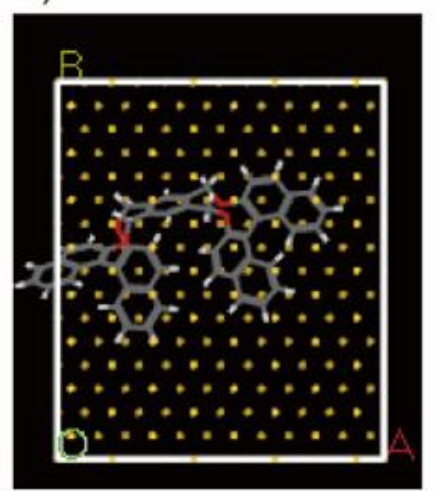

e)

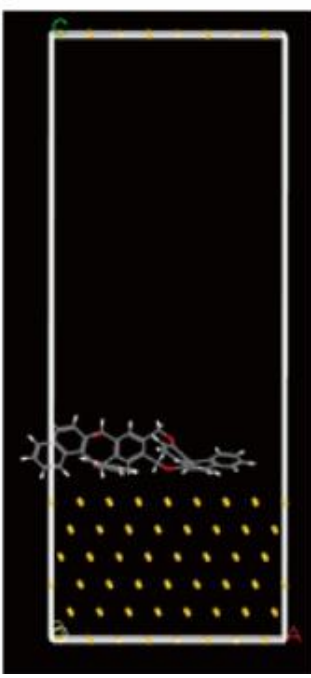

c)

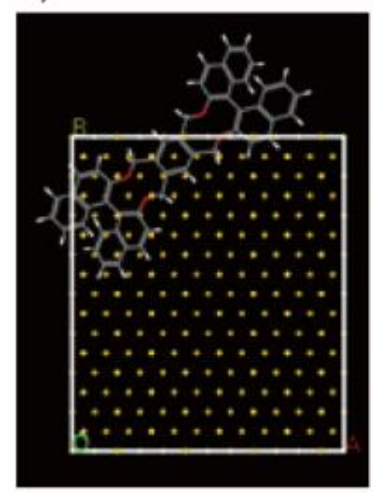

f)

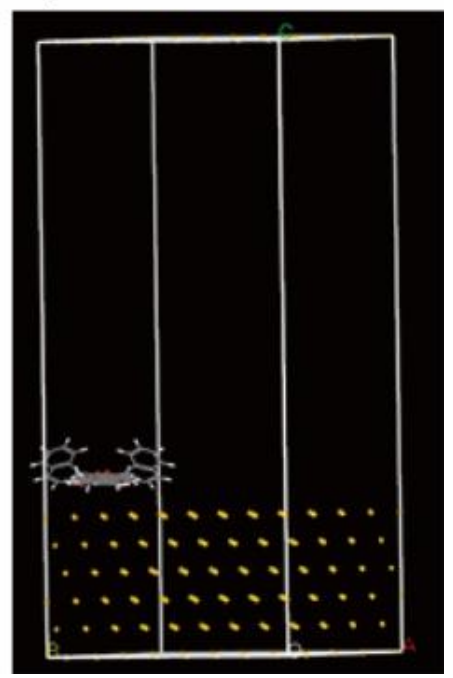

Fig. S18. $(a, d)$ anti-, $(b, e)$ syn-, $(c, f)$ flat-BBD on $A u(111)$ layers simulated by MD calculations. 


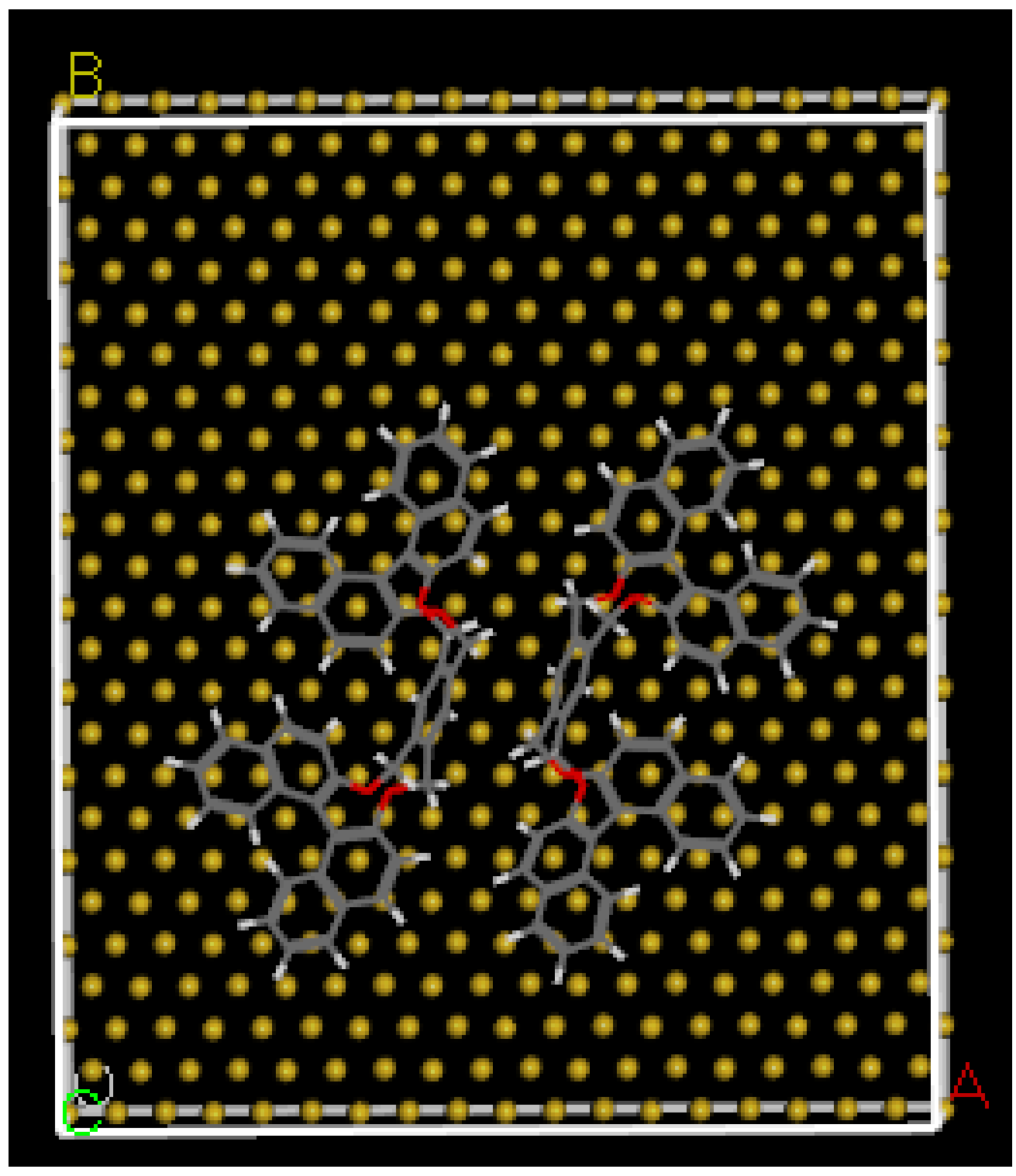

Fig. S19. syn-syn-BBDs on $A u(111)$ layers simulated by MD calculations. 

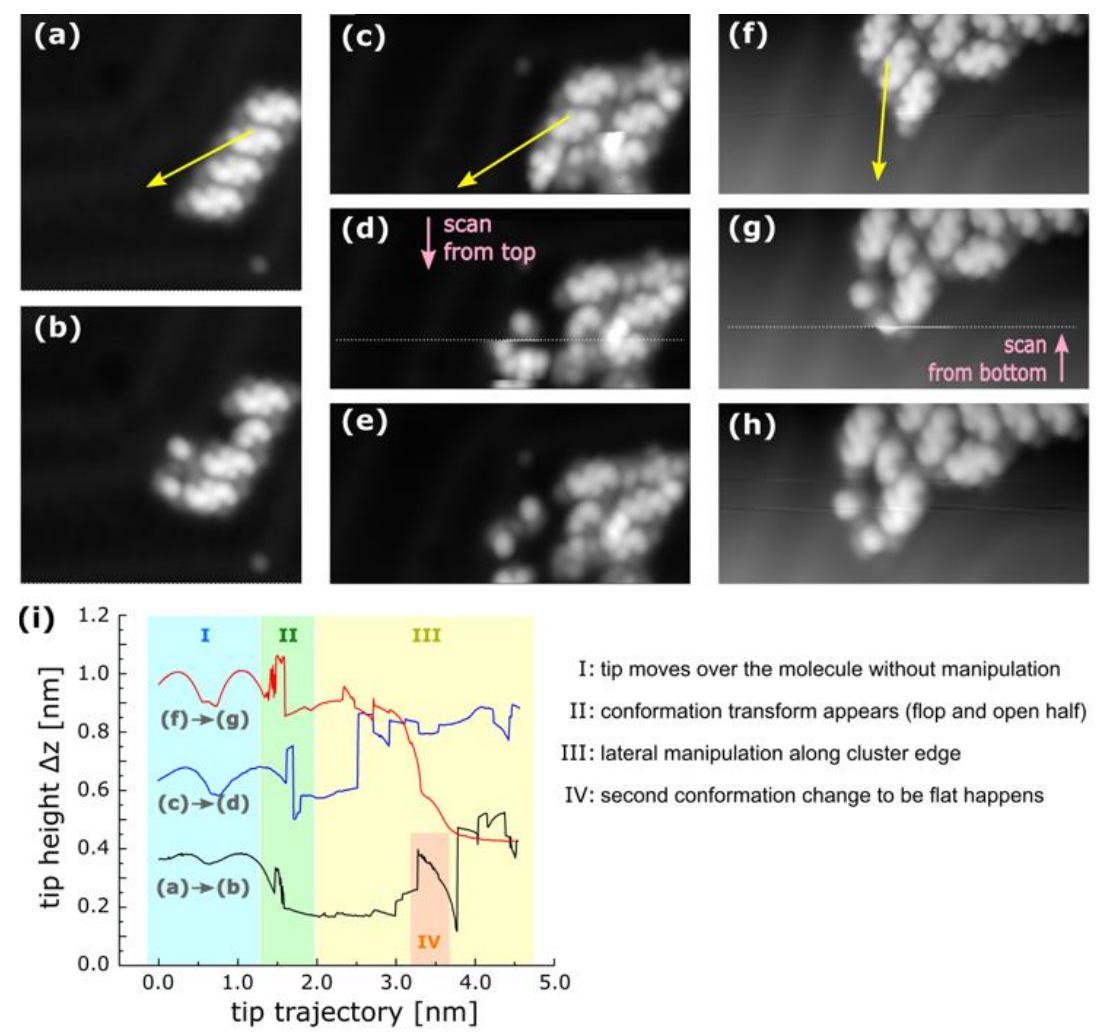

I: tip moves over the molecule without manipulation

II: conformation transform appears (flop and open half)

III: lateral manipulation along cluster edge

IV: second conformation change to be flat happens

Fig. S20. Three examples of conformation manipulation. (a)-(b) syn-to flat-conformation transformation given by single forcible manipulation. $(c)-(e)$ and $(f)-(h)$ conformation transformations via a metastable state having one paddle to be flat and other one staying syn-like form. $(d)$ and $(g)$ taken just after manipulations indicate that this metastable conformation is transformed instantly to full flat-conformation at the dotted scan lines shown in $(e)$ and $(h)$ recorded continued scan after $(d)$ and $(g)$ respectively. Here dot lines are boundaries imaging different states of molecule before and after this transformation. In the last part of scan $(d)$, another unexpected event occurs to separate unnecessary molecule from flat one. (i) tip height profiles during each manipulation and the corresponding interpretation of each manipulation signal. 
observed a clear conformation change of one of the two paddle of the BBD molecule. This change can be noticed by a different lateral extension and apparent height of the corresponding paddle (0.04 $\mathrm{nm}$ in height). The exact local transformation of this paddle molecular geometry is now under investigation. Notice that due to the stability of our LT-UHV 4 STM and to the cleanness of the STM tip apex end, this image difference is not due to a tip effect.

See Fig. S21. 

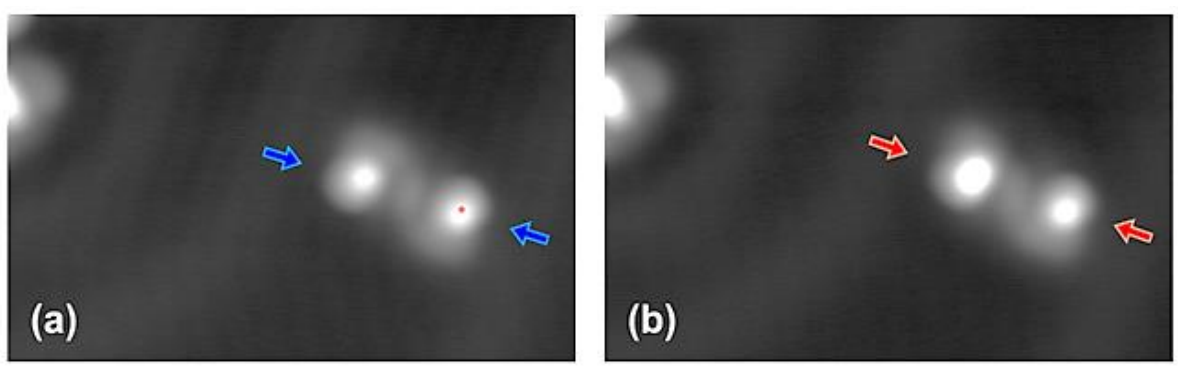

(c)

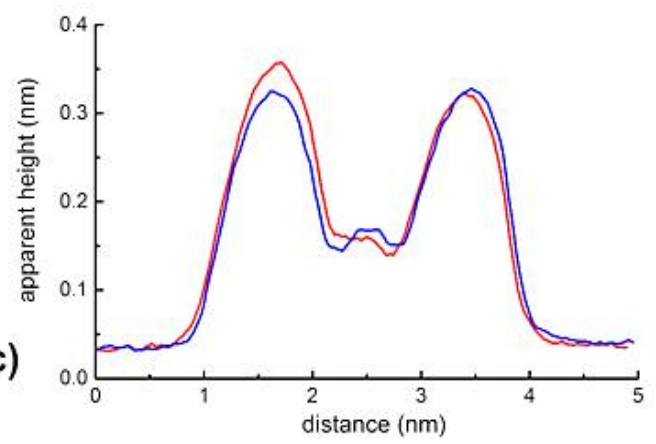

Fig. S21. The change from a flat to a syn/flat like conformation induced by inelastic electronic tunneling excitations. (a) The flat conformation of the BBD molecule before excitation. The red dot is indicating the highest electronic probability density location of first excited states of the BBD molecule where the STM tip apex was positioned during the excitation. (b) The syn/flat conformation after this excitation. The conformation change from (a) to (b) occurs opposite to the excited paddle (right paddle surrounded by yellow dotted line). (c) Line scan profiles joining the highest points of both-end paddles before (blue) and after (red) excitation. 
* E-mail: we-hyo.soe@cemes.fr

$\dagger$ Present Address: Department of Chemical Engineering, Kyushu University, 744 Motooka, Nishi-ku, Fukuoka 819-0395, Japan.

‡ E-mail: Nakanishi.Waka@nims.go.jp.

1 Frisch, M. J.; Trucks, G. W.; Schlegel, H. B.; Scuseria, G. E.; Robb, M. A.; Cheeseman, J. R.; Scalmani, G.; Barone, V.; Mennucci, B.; Petersson, G. A.; Nakatsuji, H.; Caricato, M.; Li, X.; Hratchian, H. P.; Izmaylov, A. F.; Bloino, J.; Zheng, G.; Sonnenberg, J. L.; Hada, M.; Ehara, M; Toyota, K. et al. Gaussian 09, Revision B.01; Gaussian, Inc.: Wallingford, CT, 2009.

2 Becke, A. D. Density-Functional Exchange-Energy Approximation with Correct Asymptotic Behavior. Phys. Rev. A 1988, 38, 3098-3100.

3 Becke, A. D. Density-Functional Thermochemistry. III. The Role of Exact Exchange. J. Chem. Phys. $1993,98,5648-5652$.

${ }^{4}$ Lee, C.; Yang, W.; Parr, R. G. Development of the Colle-Salvetti Correlation-Energy Formula into a Functional of the Electron Density. Phys. Rev. B 1988, 37, 785-789.

5 Ditchfield, R.; Hehre, W. J.; Pople, J. A. Self-Consistent Molecular-Orbital Methods. IX. An Extended Gaussian-Type Basis for Molecular-Orbital Studies of Organic Molecules. J. Chem. Phys. 1971, 54, 724728.

6 Hehre, W. J.; Ditchfield, R.; Pople, J. A. Self-Consistent Molecular Orbital Methods. XII. Further Extensions of Gaussian-Type Basis Sets for Use in Molecular Orbital Studies of Organic Molecules. J. Chem. Phys. 1972, 56, 2257-2261.

7 Hariharan, P. C.; Pople, J. A. Accuracy of $\mathrm{AH}_{n}$ Equilibrium Geometries by Single Determinant Molecular Orbital Theory. Mol. Phys. 1974, 27, 209-214.

8 Hariharan, P. C.; Pople, J. A. The Influence of Polarization Functions on Molecular Orbital Hydrogenation Energies. Theor. Chem. Acc. 1973, 28, 213-222.

9 Hochstrasser, R. M. The Effect of Intramolecular Twisting on the Emission Spectra of Hindered Aromatic Molecules. Can. J. Chem. 1960, 39, 459-470. 